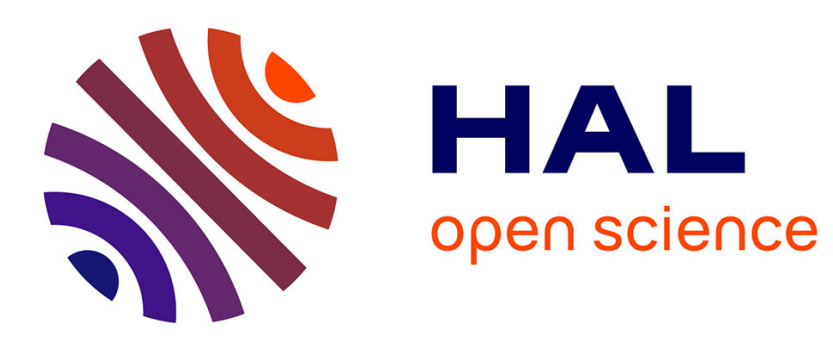

\title{
Fast detection of facial wrinkles based on Gabor features using image morphology and geometric constraints
}

\author{
Nazre Batool, Rama Chellappa
}

\section{To cite this version:}

Nazre Batool, Rama Chellappa. Fast detection of facial wrinkles based on Gabor features using image morphology and geometric constraints. Pattern Recognition, 2015, 48, pp.642 - 658 . 10.1016/j.patcog.2014.08.003 . hal-01096629

\section{HAL Id: hal-01096629 \\ https://hal.inria.fr/hal-01096629}

Submitted on 17 Dec 2014

HAL is a multi-disciplinary open access archive for the deposit and dissemination of scientific research documents, whether they are published or not. The documents may come from teaching and research institutions in France or abroad, or from public or private research centers.
L'archive ouverte pluridisciplinaire HAL, est destinée au dépôt et à la diffusion de documents scientifiques de niveau recherche, publiés ou non, émanant des établissements d'enseignement et de recherche français ou étrangers, des laboratoires publics ou privés. 


\title{
Fast Detection of Facial Wrinkles based on Gabor Features using Image Morphology and Geometric Constraints
}

\author{
Nazre Batool and Rama Chellappa \\ Department Of Electrical and Computer Engineering and the Center for Automation \\ Research, UMIACS, University of Maryland, College Park, MD 20742, USA. \\ nazr.e.batool@gmail.com,rama@umiacs.umd.edu
}

\begin{abstract}
Facial wrinkles are important features of aging human skin which can be incorporated in several image-based applications related to aging. Facial wrinkles are 3D features of skin and appear as subtle discontinuities or cracks in surrounding skin texture. However, facial wrinkles can easily be masked by illumination/acquisition conditions in $2 \mathrm{D}$ images due to the specific nature of skin surface texture and its reflective properties. Existing approaches to image-based analysis of aging skin are based on analysis of wrinkles as texture and not as curvilinear discontinuity/crack features. Previously, we proposed a stochastic approach based on Marked Point Processes (MPP) to localize facial wrinkles as curves. In this paper, we present a fast deterministic algorithm based on Gabor filters and image morphology to improve localization results. We propose image features based on Gabor filter bank to highlight the subtle curvilinear discontinuities in skin texture caused by wrinkles. Then, image morphology is used to incorporate geometric constraints to localize curvilinear shapes of wrinkles at image sites of large Gabor filter responses. Experiments are conducted on two sets of low and high resolution images and results are compared with those of MPP modeling. Experiments show that not only the proposed algorithm is significantly faster than MPPbased approach but also provides visually better results.

Keywords: Facial wrinkles, Wrinkle detection, Gabor features, Geometric constraints, Image morphology, Skin texture, Aging skin, Curvilinear object detection
\end{abstract}




\section{Introduction}

Wrinkles and fine lines are important facial features present in most aging faces. An accurate image-based analysis of these features can play an important role in relevant aging applications e.g. age estimation, age simulation and recognition across aging [1, 2, 3, 4, 5]. However, we observe that the methodologies used in these applications are mainly based on the incorporation of wrinkles as texture features and only a few attempts have been made to evaluate wrinkles as edges/curvilinear objects. For example, two pieces of work presented in $[6,7]$ analyze wrinkles as edges for the quantification of the severity of wrinkles. In general, image-based detection and localization of wrinkles as curvilinear objects has remained unaddressed. Facial wrinkles create the appearance of texture on skin but, when observed at high resolution, do not depict any repetitive/homogeneous pattern as is the defining characteristic of texture patterns. More precisely, wrinkles can best be described as subtle discontinuities, faults or cracks in surrounding inhomogeneous skin texture.

Based on this observation, previously we proposed a model based on Marked Point Processes (MPP) to localize wrinkles as line segments/curves [8, 9]. This work was actually inspired by an early work by Stoica et al. [10] in which MPP modeling was used to incorporate geometric constraints for the detection of roads as curvilinear objects in aerial images. Following a similar approach, we modeled wrinkles as a spatial process of line segments in a Bayesian framework. The prior probability model was used to incorporate known spatial characteristics of facial wrinkles to distinguish edges due to wrinkles from those due to other factors. Then the facial wrinkles were detected by sampling the posterior distribution using Reversible Jump Markov Chain Monte Carlo (RJMCMC) algorithm. Although based on the prior model, edges due to wrinkles were well distinguished from erroneous ones, the simulation using RJMCMC algorithm brought to light some limitations. First, the value of the parameter $\alpha$ varied with image resolution. Second, the simulated annealing procedure used for global optimization required a slower cooling schedule to be effective. Finally, a large number of iterations was needed for the RJMCMC algorithm requiring considerable computation time. These factors led us to adopt a deterministic approach as compared to the stochastic approach of MPP modeling. Furthermore, the experiments

underlined some of the specific challenges in localization of wrinkles due to the nature of skin imaging. It was observed that skin features other than 
wrinkles (e.g. pores and brown spots) cause significant intensity gradients in $2 \mathrm{D}$ images. This poses a problem, specifically to the detection of light wrinkles which appear as very subtle discontinuities in surrounding skin texture. In such cases, intensity gradients due to light wrinkles are less obvious as compared to those due to other factors. Another challenge is the drastically varying skin appearance in images with variations in illumination and acquisition angles [11]. The specific texture of skin causes bright spots masking intensity gradients due to wrinkles.

Facial wrinkles localized as curvilinear objects can be used in several applications. For example, we used automatically localized as well as handdrawn wrinkles to investigate the discriminative power of wrinkle patterns as soft biometrics [12]. In another application, localized wrinkles were removed via image inpainting using a constrained textre synthesis technique. This work was proposed as an advancement to the current facial retrouching or skin beautification applications [13].

Although signal processing techniques such as wavelet transforms, shearlet transforms, Gabor filters, etc. can be applied to enhance the intensity gradients caused by wrinkles in color/gray scale images, these methods have limitations. The specific granular skin surface texture and variable skin tone in addition to illumination give rise to large intensity gradients and false positives in both blob-like and curvilinear shapes. Accurate localization of facial wrinkles will involve eliminating such false positives and separating deep to light intensity gradients caused by wrinkles from those caused by other factors. In the proposed algorithm, we first use a Gabor filter bank with specific parameter values to highlight curvilinear features. Then, we use image morphological techniques, instead of simple thresholding, to place curves at image sites of large Gabor responses.

The main contributions of this work are as follows:

1. We present an algorithm which can localize subtle discontinuities/cracks in skin texture caused by wrinkles as curvilinear objects. It is hoped that such explicit localization of wrinkles will aid several applications related to aging skin, facial biometrics, recognition across aging and monitoring of skin in future.

2. We present a fast deterministic algorithm which is several times faster than the previously proposed approach based on Marked Point Processes. In addition, the algorithm also improves detection results significantly. 
3. We introduce a dataset of around 125 high resolution images of public figures downloaded from the Internet along with hand-drawn wrinkles. We conducted experiments on this dataset in addition to a subset of FG-NET database and the dataset used in [8, 9].

4. We introduce an elaborate algorithm based on mathematical morphology to place curvilinear objects at sites of high intensity gradients and to reduce false positives.

5. From a detailed analysis of experimental results, we highlight typical challenges in the localization of wrinkles in 2D images.

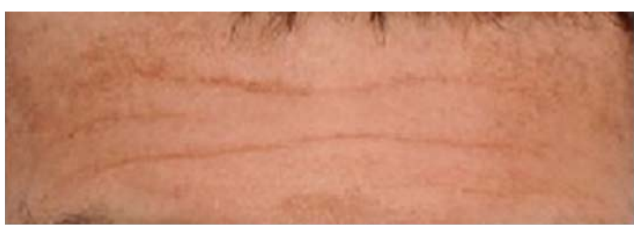

(a)

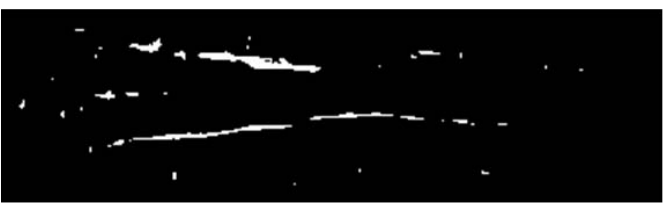

(c)

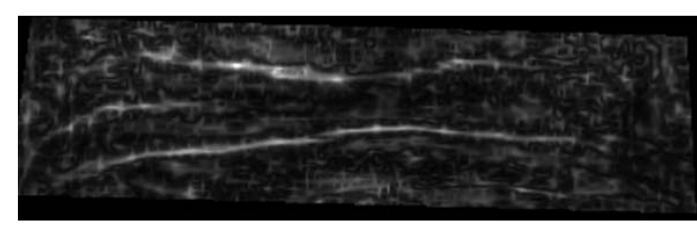

(b)

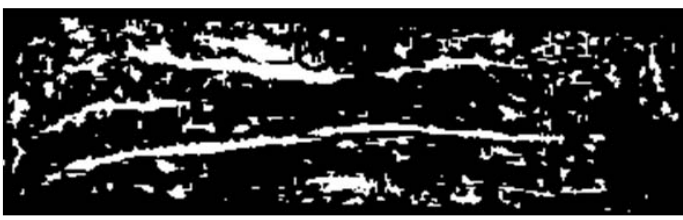

(d)

Figure 1: A simple thresholding of Gabor image features. (a) Original forehead image. (b) Gabor image features scaled to gray scale. (c) Resulting binary image with threshold value of 0.5 . (d) Resulting binary image with threshold value of 0.3 .

\section{Related Work}

Curvilinear structures of interest appear in several types of medical and non-medical images. These structures can either constitute main objects in an image or can appear as some sort of anomaly/discontinuity in the main objects of an image. Some examples of the former case are finger prints in finger print images, retinal blood vessels in fundus camera images and brain sulcal lines in MRI scan images. Some examples of the latter are the structures like buildings, roads and rivers in aerial images and cracks in manufactured steel slabs and railway tracks. An extensive survey of all the related work regarding the detection of curvilinear structures is out of scope 
of this paper. Here, we review methods based on filtering and mathematical morphology.

Normally, the targeted curvilinear structures have image features very similar to those of background making an accurate extraction of their shapes a challenging problem. A thorough survey of detection of cracks in roads can be found in [14] and detection of blood vessels in retinal images can be found in [15]. In cases where enough data is available, a viable approach is to apply machine learning techniques to learn an appropriate data model. The other possible approach is to use filtering (e.g. Gabor filters, Wavelets, Contourlets, Gaussian filters) and/or morphological processing of the data. Several oriented feature detectors have been developed including steerable Gaussian second-derivative filters, line operators and Gabor filters. A comparative study can be found in [16] where the real Gabor filters were assessed to be the best detector of oriented features. However, a simple thresholding of such filter responses is usually unable to yield well localized wrinkle curves. As an example, Figure 1 depicts a forehead image and the corresponding maximum amplitude filtered response from a Gabor filter bank (details in Section 3.1) as a gray scale image. It can be observed that wrinkle discontinuities are well highlighted by a Gabor filter bank but, as seen in Figure 1(c) and (d), are not well localized as curves by simple thresholding. Hence, we apply morphological techniques to retrieve wrinkle curves from thresholded Gabor response images.

In cases where prior knowledge about the geometric properties of the objects of interest is available, mathematical morphology can be a powerful tool to enhance and/or identify such objects. Consequently, morphology has been used as segmentation and post-processing technique in detecting curvilinear structures in wide range of applications. An early piece of work on detection of (blood-vessel like) curvilinear structures in medical images using morphology was presented by Zana and Klein in [17]. The so-called vessellike patterns were defined to have certain geometric properties of shape, width, connectivity and separation. These properties were manipulated by the morphological operations of top-hat, opening and closing to highlight such patterns to be analyzed further for their curvature. Landstrom and Thurley proposed an algorithm based on several morphological operations to detect cracks in steel slabs from 3D range data [18] while focusing on minimizing false positives. Lam and Hong Yan used divergence of vector fields to detect vessel like objects in retinal images and then applied skeletization to remove artifacts [19]. Chambon and Moliard used locally variant 
thresholding, closing, median/mean filtering and histogram equalization to detect cracks in road pavements [14]. Lelore and Bouchara used dilation and analysis of pixel-level neighborhoods to reduce false positives in document image restoration [20]. In the absence of large training data, we follow these examples and adopt a morphological approach to incorporate the geometric properties of facial wrinkles and localize wrinkles as curvilinear objects.

\section{Approach}

As mentioned earlier, the MPP model provided a way to incorporate prior knowledge about wrinkles in the form of spatial interaction of line segments. For example, the overlapping and congestion of line segments were penalized. Since such spatial interactive rules were a key to distinguishing edges due to wrinkles from those due to other factors, a deterministic approach incorporating similar rules is expected to perform better for the detection of wrinkles. In this work, we apply gradual thresholding and morphological processing to incorporate geometric characteristics of facial wrinkles. The resulting geometric constraints can then discriminate between image intensity gradients due to wrinkles and other factors. Our approach is based on the following assumptions regarding geometrical and image characteristics of wrinkles.

A1: Wrinkles cause intensity gradients which are highlighted by Gabor filter bank.

A2: Wrinkles appear as curvilinear features instead of blob like features.

A3: Wrinkles are continuous and not a series of disconnected curvilinear segments.

A4: Wrinkles are less probable to intersect each other or to be congested in small areas.

\subsection{Gabor Filters and Image Features}

We use Gabor filter responses as image features to highlight the curvilin-

ear features of wrinkles in this work. The real Gabor filter kernel oriented at the angle $\alpha$ is given by

$$
g\left(x_{1}, x_{2}\right)=\frac{1}{2 \pi \sigma_{x_{1}} \sigma_{x_{2}}} \exp \left[\frac{-1}{2}\left(\frac{x_{1}^{\prime 2}}{\sigma_{x_{1}}^{2}}+\frac{\gamma^{2} x_{2}^{\prime 2}}{\sigma_{x_{2}}^{2}}\right)\right] \cos \left(2 \pi f x_{1}^{\prime}\right)
$$


where the parameters $\sigma_{x_{1}}, \sigma_{x_{2}}$ denote the scale of the 2D Gaussian envelope, $f$ denotes the frequency of the sinusoid and $\gamma$ denotes the spatial aspect ratio which defines the ellipticity or the elongation of support of Gabor function. The values for $x_{1}, x_{2}$ are given as follows:

$$
\left[\begin{array}{l}
x_{1}^{\prime} \\
x_{2}^{\prime}
\end{array}\right]=\left[\begin{array}{cc}
\cos \alpha & \sin \alpha \\
-\sin \alpha & \cos \alpha
\end{array}\right]\left[\begin{array}{l}
x_{1} \\
x_{2}
\end{array}\right]
$$

Let $\left\{g_{k}\left(x_{1}, x_{2}\right), k=0, \cdots, K-1\right\}$ denote the set of real Gabor filters oriented at angles $\alpha_{k}=-\frac{\pi}{2}+\frac{\pi k}{K}$ where $K$ is the total number of equally spaced filters over the angular range $\left[\frac{-\pi}{2}, \frac{\pi}{2}\right]$. Let $\left\{I\left(x_{1}, x_{2}\right) ; x_{1}=1 \ldots N_{1}, x_{2}=1 \ldots N_{2}\right\}$ denote the input image in gray scale and $I_{k}^{F}\left(x_{1}, x_{2}\right)$ denote the image filtered by the filter $g_{k}\left(x_{1}, x_{2}\right)$. The corresponding maximum amplitude among the filtered responses is given as:

$$
I^{\prime}\left(x_{1}, x_{2}\right)=\max _{k} I_{k}^{F}\left(x_{1}, x_{2}\right)
$$

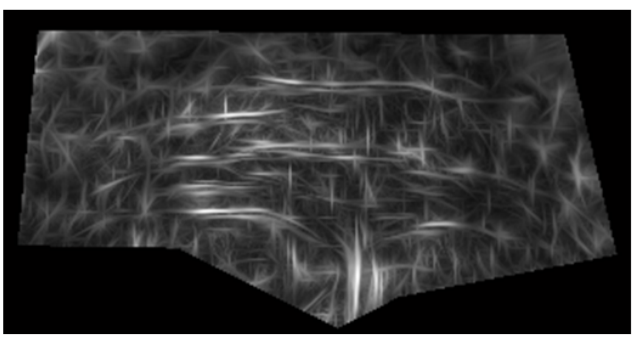

(a)

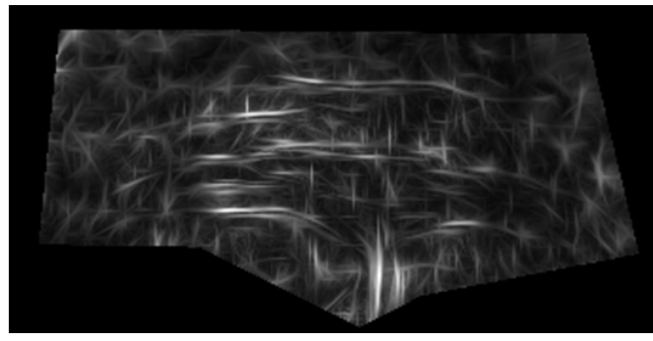

(b)

Figure 2: Values of $I^{N}\left(x_{1}, x_{2}\right)$ (equation 4) in gray scale, (a) $p=1$ (b) $p=1.25$. Highlighting of image sites with larger Gabor responses can be observed in (b).

The maximum amplitude response is normalized to the range $[0,1]$ according to the following equations:

$$
\begin{gathered}
I^{\prime \prime}\left(x_{1}, x_{2}\right)=\left(I^{\prime}\left(x_{1}, x_{2}\right)-\min _{\left(x_{1}, x_{2}\right)} I^{\prime}\left(x_{1}, x_{2}\right)\right)^{p} \\
I^{N}\left(x_{1}, x_{2}\right)=\frac{I^{\prime \prime}\left(x_{1}, x_{2}\right)}{\max _{\left(x_{1}, x_{2}\right)} I^{\prime \prime}\left(x_{1}, x_{2}\right)}
\end{gathered}
$$

where the value of the parameter $p$ is set to be greater than 1 to highlight the image sites with larger Gabor filter responses. Figure 2 includes two 


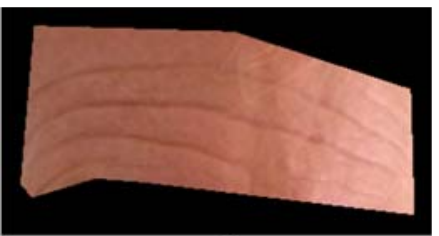

(a)

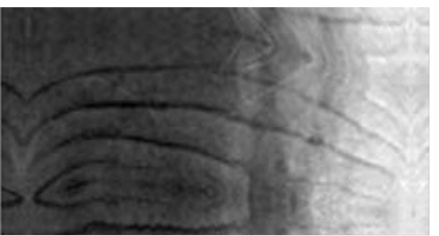

(b)

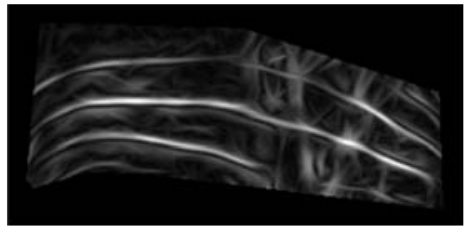

(c)

Figure 3: Boundary conditions to calculate Gabor features. (a) User-cropped forehead image embedded in a rectangular region with a margin. (b) Gray scale image with mirror boundaries. (c) Gabor image features scaled to gray scale.

images of $I^{N}\left(x_{1}, x_{2}\right)$ with $p=1$ and $p=1.25$ as an example. The set of the normalized maximum filter response , $\left\{I^{N}\left(x_{1}, x_{2}\right)\right\}$, constitutes image features for automatic detection of wrinkles. Figure 3(a) shows a user-cropped forehead image embedded in a rectangular image. Figure 3(b) depicts the mirror boundary conditions used to fill the black border around the forehead image in Figure 3(a) to eliminate the boundary artifacts in Gabor response images. Figure 3(c) shows the corresponding maximum Gabor responses in the original forehead image. It can be observed that high filter responses are caused by illumination variations, appearing mostly vertically in the middle of the forehead, and wrinkles.

\subsection{The Algorithm}

Figure 4 shows the block diagram for our three-step algorithm. The first step is based on assumptions 'A1' and 'A2' and aims at detecting key image sites with high gradients probably caused by wrinkles than by other factors. This is done by gradual thresholding and discarding of the thresholded bloblike regions. The second step is based on assumptions 'A3' and 'A4'. The purpose of this step is to trace wrinkle curves out from key image sites detected in the first step. Wrinkle curves are extended from key image sites while penalizing congestion or intersection of wrinkle curves. The third step reduces false positives in the binary output image of the last two steps. We now present the details of incorporating the geometric constraints in the proposed three-step algorithm.

\subsection{Step 'A': Detection of Key Wrinkle Sites}

This step is based on the first two assumptions that wrinkles are curvilinear structures and cause high intensity gradients in the image. In this step, 


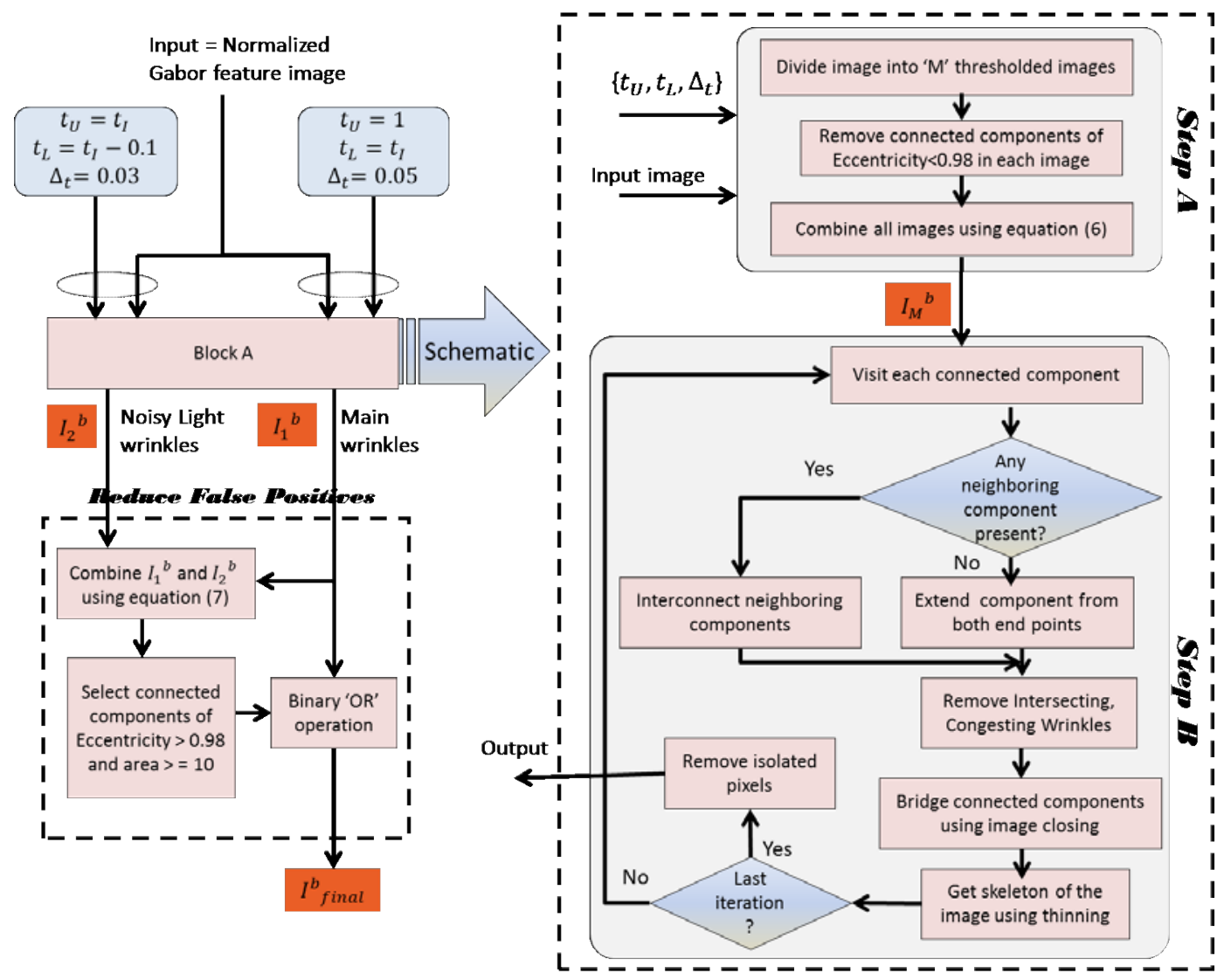

Figure 4: Block diagram of wrinkle localization algorithm.

non-overlapping clusters of image sites with high Gabor filter responses and in curvilinear shapes (instead of blob-like shapes) are detected. Such sites have higher probability of being part of a wrinkle. The is similar to the placement of seed segments under MPP modeling where a favorable initial state in the RJMCMC algorithm was provided by placing 'seed' segments on image sites with the highest LoG filter responses. For the detection of key wrinkle sites, successive thresholding of the image $I^{N}\left(x_{1}, x_{2}\right)$ and morphological processing of the resulting binary images are applied.

Let $T=\left\{t_{m} \mid t_{m}=t_{U}-m \Delta_{t}, m=1,2, \cdots, M ; t_{L}, t_{U} \in[0,1]\right\}$ denote a set of thresholds. The image $I^{N}\left(x_{1}, x_{2}\right)$ is quantized in threshold increments of $\Delta_{t}$ resulting in $M$ binary images where the number $M$ is determined by the selection of $t_{L}$ and $\Delta_{t}$. Let each binary image be denoted as $i_{m}$ which is processed to discard the connected components having blob-like and non- 


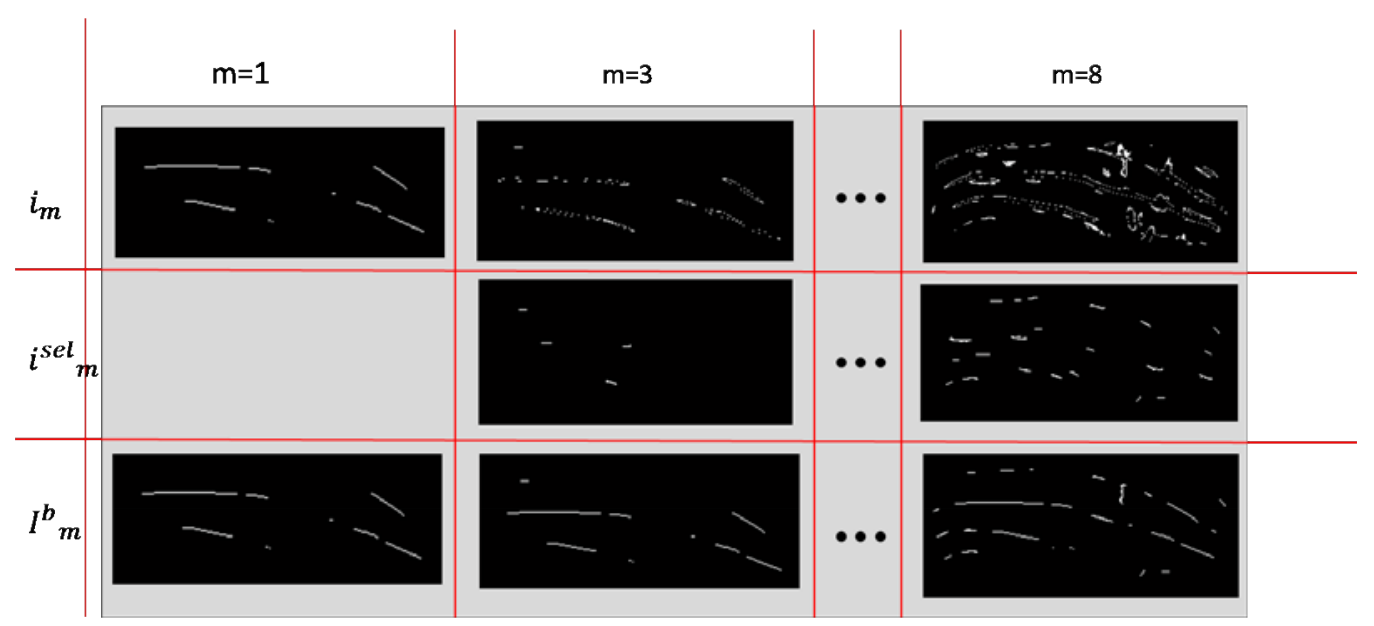

Figure 5: Detection of key wrinkle sites in step 'A' of the algorithm.

curvilinear shapes using the 'eccentricity' property of connected components. Let $i_{m}^{s e l}$ denote the resulting binary images after removal of blob-like components and having only curvilinear portions. The binary images are then combined while keeping a certain distance among selected connected components. The reason for maintaining a distance is to prevent consecutively thresholded connected components from aggregating into non-linear shapes which would normally result from a simple thresholding of Gabor response image. Let us assume that we want to select connected components $r^{d}$ pixels apart. Then the images are dilated by a disk, denoted by $D_{r^{d}}$, of radius $r^{d}$ and combined using 'OR' operation recursively to obtain the final key wrinkle sites according to the following equations:

$$
\begin{aligned}
I_{1}^{b} & =i_{1}^{\text {sel }} \\
I_{2}^{b} & =\left(I_{1}^{b} \oplus_{b} D_{r^{d}}\right)^{c} \vee i_{2}^{\text {sel }} \\
\vdots & \\
I_{M}^{b} & =\left(I_{M-1}^{b} \oplus_{b} D_{r^{d}}\right)^{c} \vee i_{M}^{\text {sel }}
\end{aligned}
$$

where symbols ' $\oplus_{b}$ ', ' $(\cdots)^{c}$ ' and ' $\mathrm{V}$ ' denote the operations of binary dilation, 'NOT' and 'OR' respectively. Figure 5 illustrates this process where the top row shows simple thresholded binary images with several connected components at three different iterations. The middle row shows images after discarding connected components of high eccentricity values. The bottom 
row shows the combined binary image up to that iteration. The final image, $I_{M}^{b}$, consists of key wrinkle sites and is processed in the second step of the algorithm.

\subsection{Step 'B': Tracing Wrinkle Curves from Key Wrinkle Sites}

After the key sites have been detected, the next step is to trace wrinkle curves from those sites. This step is similar to the birth and death moves of line segments in the MPP model where new line segments are connected to the existing ones. The processing in this step is based on the assumptions 'A2', 'A3' and 'A4' regarding the geometric characteristics of wrinkles.

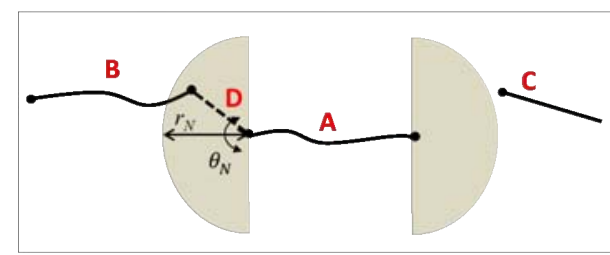

(a)

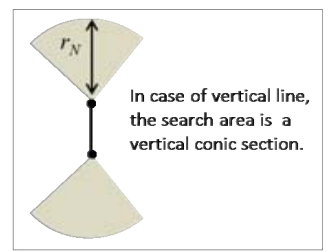

(b)

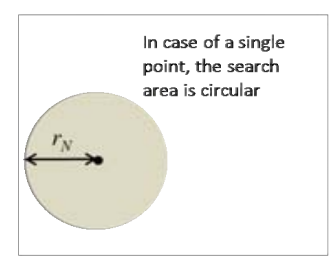

(c)

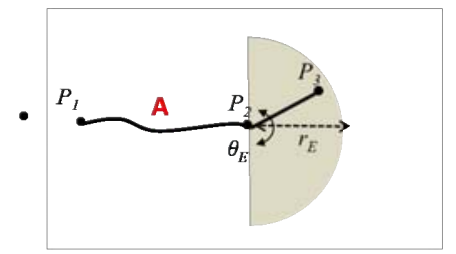

(d)

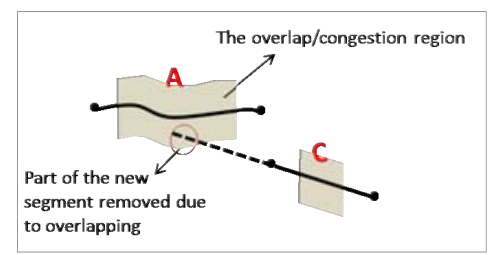

(e)

Figure 6: Graphical illustration of the geometric constraints of the connected components. (a) 'A' is connected to 'B' through ' $D$ ' as 'B' lies in the conic search area of 'A'. (b) Search region for a vertical line (c) Search region for a single point (d) 'A' is extended beyond site $P_{2}$ by including $\overline{P_{2} P_{3}}$. Site $P_{3}$ has the maximum Gabor response in the conic search area. (e) Penalizing congestion/intersection of connected components.

\subsubsection{Continuity of Wrinkles}

The third geometrical property of wrinkles, continuity, is incorporated in two steps. First, a search area of a certain shape, based on the orientation of the connected component, is searched around each end point of the connected component. If there exists an end point of a neighboring connected component, the two components are joined. Figure 6(a) demonstrates this step. The component ' $\mathrm{A}$ ' has an end point of a nearby component ' $\mathrm{B}$ ' in the search area at its left end point. Hence, the two components are joined by 
placing the component ' $\mathrm{D}$ ' between their end points. Regarding the search area of the right end point of the component 'A', since there is no end point of any other component, this end point will be extended in the next step. The shape of search area depends on the orientation of the connected component to ensure smoothly varying wrinkle curves. The search area is a circular region in case of a single point component (Figure 6(b)), vertically oriented conic regions in case of a component as vertical line (Figure 6(c)) and horizontally oriented conic regions otherwise (Figure 6(a)).

If no neighboring components are present, for continuity, the end points of a connected component are extended to nearby Gabor response maxima. As an example, in Figure 6(d), the component ' $\mathrm{A}$ ' is extended from the right end point $P_{2}$. First a conic region of the radius $r_{E}$ and angle $\theta_{E}$ is searched for the maximum $I^{N}\left(x_{1}, x_{2}\right)$. If this maximum is greater than $t_{L}$, the corresponding point is considered for extension. Let this point be denoted as $P_{3}$ in Figure 6(d). The component ' $\mathrm{A}$ ' is then extended by adding line segment $\overline{P_{2} P_{3}}$. This process can be considered similar to the active contours (or 'snake') algorithms where an initial curvilinear segment is drifted to the local maxima along the directions of local gradients. However, in case of wrinkles, a simpler extension suffices because the newer extended segments are only a few pixels long and a greedy algorithm is efficient.

As is shown in Figure 4, this process is repeated for many iterations where, in each iteration, connected segments are visited and extended to give curvilinear objects. The extensions eventually stop when there are no neighboring sites with $I^{N}\left(x_{1}, x_{2}\right)>t_{L}$. We used 30 iterations in our experiments.

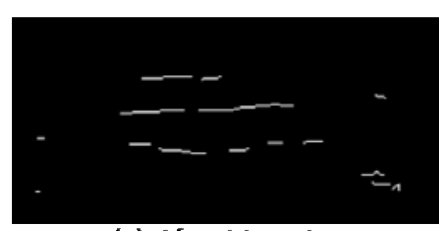

(a) After 1 iteration

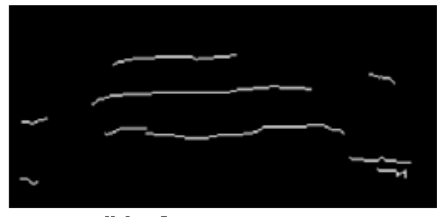

(b) After 6 iterations

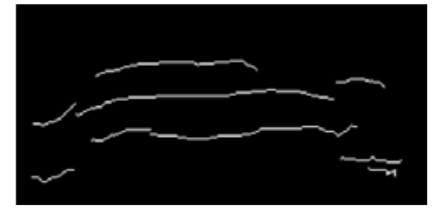

(c) After 13 iterations

Figure 7: Step 'B': Extension of wrinkle curves from key wrinkle sites.

\subsubsection{Penalizing Intersection/Congestion of Wrinkles}

In the process of tracing wrinkle curves from key wrinkle sites, the assumption 'A4' is incorporated to penalize intersection and congestion of wrinkle curves. This is achieved using binary dilation. A non-overlapping area 
for every connected component is obtained by dilating it with a rectangular region. During extension and drifting of wrinkle curves, any portions overlapping with these areas are discarded. As an example, Figure 6(e) shows two wrinkle curves with their non-overlapping neighborhood. When wrinkle curve ' $\mathrm{C}$ ' is extended, its portion overlapping with the neighborhood of wrinkle curve ' $\mathrm{A}$ ' is discarded.

\subsection{Reducing False Positives}

One of the main challenges in detection is to separate light intensity gradients due to very light wrinkles from those due to rough skin texture/other factors. As we will see in experiments, this is why it is easier to localize wrinkles in smooth skin texture than in rough texture. Figure 8 includes Gabor feature images for two subjects where wrinkle curves are more prominent for smoother skin surface texture but have a lot of noise for rougher skin surface texture at low intensity gradients. Thus, this part of the algorithm aims at eliminating false positives at very low intensity gradient values. Consequently, as will be seen in experimental results, this processing step reduces false alarm ratio to an average of $0.1 \%$, much lower than that of MPP modeling.
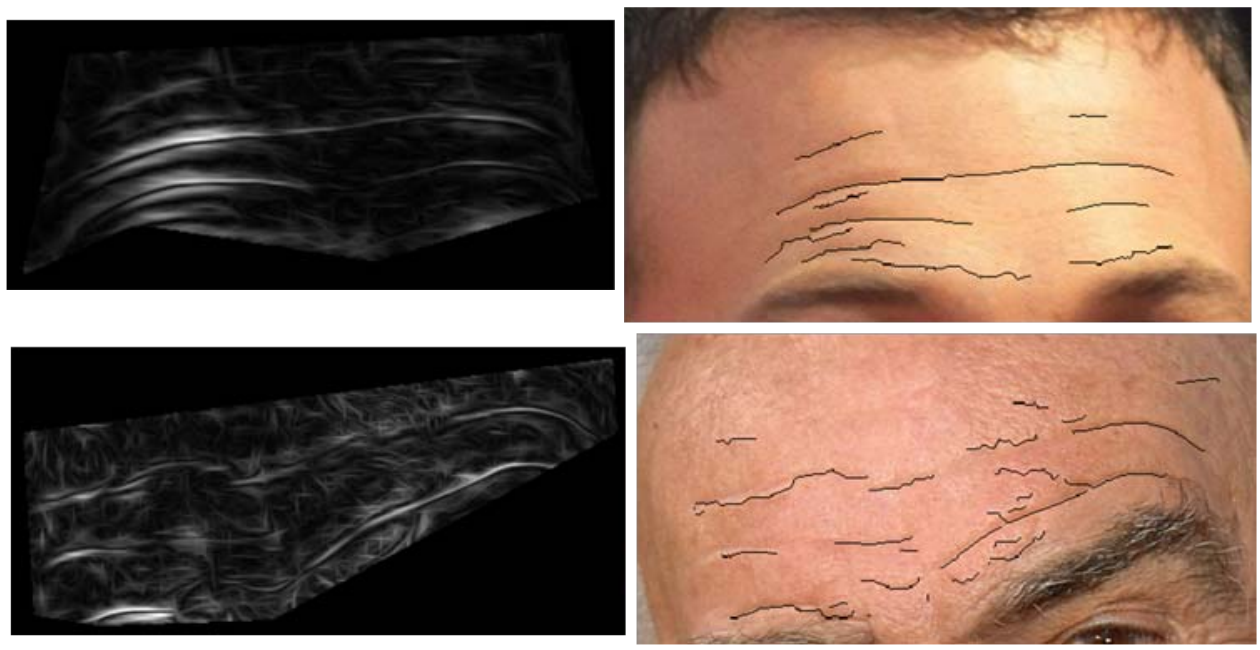

Figure 8: Gabor feature images and localized wrinkles for two subjects. (Top) Results for subject with smooth skin texture. (Bottom) Results for subject with rough skin texture.

This step is based on the assumption that the curvilinear objects detected by the steps ' $\mathrm{A}$ ' and ' $\mathrm{B}$ ' of the algorithm are more probable to be due to 


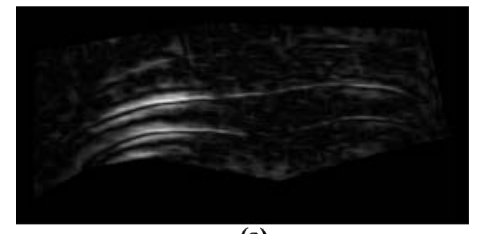

(a)

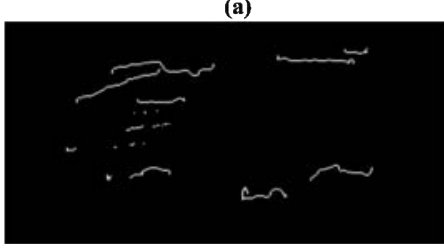

(d)

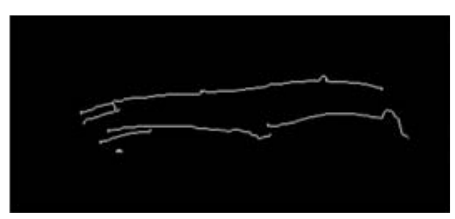

(b)

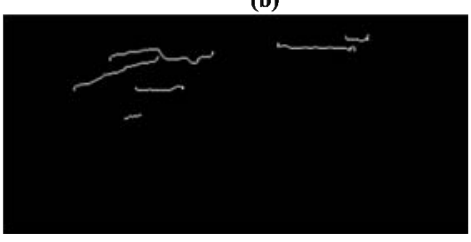

(e)

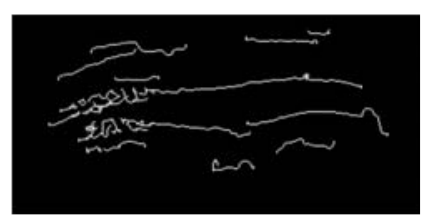

(c)

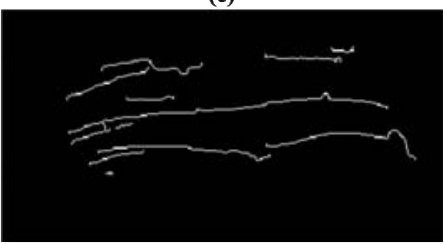

(f)

Figure 9: Minimizing false positives. (a) Gabor feature image in gray scale. (b) $I_{1}^{b}$ (c) $I_{2}^{b}$ (d) $I_{2}^{b}$ After removing components overlapping with dilated $I_{1}^{b}$ i.e. $\left(\left(I_{1}^{b} \oplus_{b} D_{r^{d}}\right)^{c} \vee I_{2}^{b}\right)$. (e) After removing small and blob-like connected components. (f) $I_{\text {final }}^{b}$ by combining (b) and (e).

wrinkles at higher thresholds. However, at lower thresholds, the curvilinear objects are more probable to include noise/false positives as well. As is shown in Figure 4, two sets of thresholds, $\left\{t_{U}=1, t_{L}=t_{I}, \Delta_{t}=0.05\right\}$ and $\left\{t_{U}=t_{I}, t_{L}=t_{I}-0.1, \Delta_{t}=0.03\right\}$, are input to the steps 'A' and 'B', where $\left\{t_{U}, t_{L}, \Delta_{t}\right\}$ are as defined in section 3.3. Let $I_{1}^{b}$ and $I_{2}^{b}$ denote the two output binary images containing curvilinear objects obtained after processing the Gabor image with two threshold sets via steps 'A' and 'B'. Again, incorporating assumptions 'A2' and 'A3', we minimize false positives in $I_{2}^{b}$ as follows:

1. Since wrinkles are continuous, any connected components with less area are less likely to be wrinkles and discarded.

2. Since wrinkles are curvilinear, any blob-like connected components with large eccentricity values are less likely to be wrinkles and discarded.

The final binary image containing wrinkles curves is obtained as follows:

$$
I_{\text {final }}^{b}=I_{1}^{b} \vee\left(\left(I_{1}^{b} \oplus_{b} D_{r^{d}}\right)^{c} \vee I_{2}^{b}\right)^{s e l 2}
$$

where $(\cdots)^{\text {sel2 }}$ denotes the selection of connected components with the area of $\geq 10$ pixels and eccentricity of $\geq 0.98$. 


\section{Results and Discussion}

\subsection{Experiments and Parameter Settings}

We conducted experiments on two sets of images. The first set consisted of high resolution images of public figures downloaded from the Internet. The second set consisted of the low resolution images used in our previous work [9] for comparison. Facial wrinkles create a wide variety of image gradients and can vary from being very light to very deep across individuals even of the same age. For example, Figure 10 contains images of three public figures taken at the age of 52 illustrating the differences in wrinkle length and depth. At the same time, depth of wrinkles can vary significantly within one individual's face as well. Figure 11 depicts an image of a subject where wrinkles around eyes and corners of mouth are much deeper than those on forehead. For this reason we used a larger Gabor filter bank with two scales, 0.1 and 0.3 . The frequency $f$ was larger for low resolution images as compared to medium resolution images. The aspect ratio was set to be 10 to highlight elongated curvilinear features. Table 1 shows the parameter values for the two sets of images. We used images of public figures, mostly celebrities, downloaded from the Internet as our data set for the reason that medium to high resolution images of celebrities were more easily available. We also made the interesting observation that it was relatively more difficult to find images of female celebrities with facial wrinkles because of the masking of wrinkles due to skin treatments, photo retouching or occluding hair styles. We did not conduct main experiments on images from currently available aging databases e.g. FG-Net, FERET, MORPH, due to the low resolution and/or quality of images.

\begin{tabular}{|c||c|c|}
\hline Parameter & High Resolution Images & Low Resolution Images \\
\hline \hline Gabor filter frequency, $f$ & 1 & $\{5,10\}$ \\
\hline Gabor filter scale $\sigma_{x_{1}}=\sigma_{x_{2}}$ & $\{0.1,0.3\}$ & $\{0.1,0.3\}$ \\
\hline Gabor filter aspect ratio $\gamma$ & 10 & 10 \\
\hline$r^{d}$ (pixels) & 10 & 7 \\
\hline$r_{N}$ (pixels) & 6 & $r_{N}-2$ \\
\hline$r_{E}$ (pixels) & $r_{N}-2$ & $\pi / 2$ \\
\hline$\theta_{N}$ & $\pi / 2$ & $0.9 * \theta_{N}$ \\
\hline$\theta_{E}$ & $0.9 * \theta_{N}$ & \\
\hline
\end{tabular}

Table 1: Parameter values for experiments on two sets of images. 


\subsubsection{Effect of Quantization}

The set of threshold parameters $\left\{t_{U}, t_{L}, t_{I}, \Delta_{t}\right\}$ determine the level of quantization and which sites are selected in main wrinkles image $I_{1}^{b}$ vs. noisy wrinkles image $I_{2}^{b}$. The parameter $t_{U}$ does not affect the detection results as long as it is close to 1 . The values of the two parameters $\left\{t_{I}, \Delta_{t}\right\}$ influence the detection results more significantly than other parameters. $t_{I}$ is the only parameter which has to be adjusted from image to image based on the depth of wrinkles. The value of the parameter $t_{I}$ was selected to be in the set $\{0.8,0.7, \cdots, 0.2\}$. Since each subject had different depth of wrinkles, $t_{I}$ could not be selected to be constant for all images. A larger value of $t_{I}$ gave better results for the deep wrinkles and a lower value gave better results for the light wrinkles. Here we examine the effect of the parameter $\Delta_{t}$ on detection results. Selecting a low value of $\Delta_{t}$ i.e. 0.01 gives a larger number, $M$, of binary images where each image has key wrinkle sites $r^{d}$ apart. This results in more connected components of smaller area which are farther apart. In some cases, this results in missing some wrinkle sites and decreases the detection ratio but does not increase the false alarm ratio. On the other hand selecting a high value of $\Delta_{t}$ i.e. 0.09 gives a lower number, $M$, of binary images where each image has connected components of larger area and somewhat congested. In most images, this resulted in the detection of more wrinkle sites icreasing both detection and false alarm ratios. For this reason we selected the values of 0.05 and 0.03 for $\Delta_{t}$ for a good trade off between the two extreme cases. In Figure 12 we show the detection results for different values of $\Delta_{t}$ for two images. The image ' $\mathrm{A}$ ' has deep wrinkles and detection rate of greater than $70 \%$ whereas the image ' $\mathrm{B}$ ' has light wrinkles and detection rate of $61 \%$. Rows 'A1' and 'B1' illustrate detection results of key image sites $I_{1}^{b}$ and rows 'A2' and 'B2' illustrate final detection results. It can be observed that although there is some difference between key wrinkle sites corresponding to $\Delta_{t}=0.01$ and $\Delta_{t}=0.05$, the final detection results are the same. Wherease the number of key wrinkle sites and final wrinkles are significantly increased for $\Delta_{t}=0.09$ in (c).

\subsection{Evaluation Setup and Quantitative vs. Qualitative Results}

Since localization of wrinkles is a new application in image-based analysis of aging skin, we proposed a quantitative evaluation setup to assess the performance of detection algorithms in our previous work. The reader is referred to [8] for a detailed description of the evaluation setup. The terms 'detected', 'original' and 'well-localized' wrinkles are defined where the detected wrinkles 

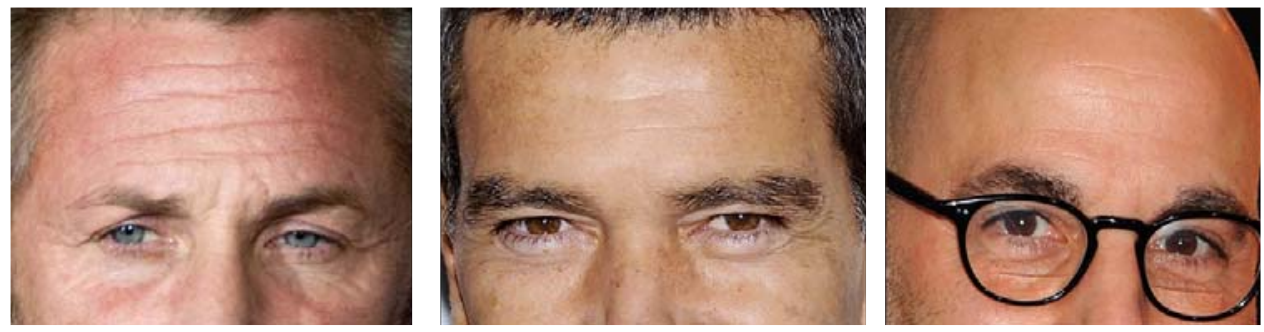

Figure 10: Three subjects of same age (52 years) have visible differences in the appearance of their wrinkles.

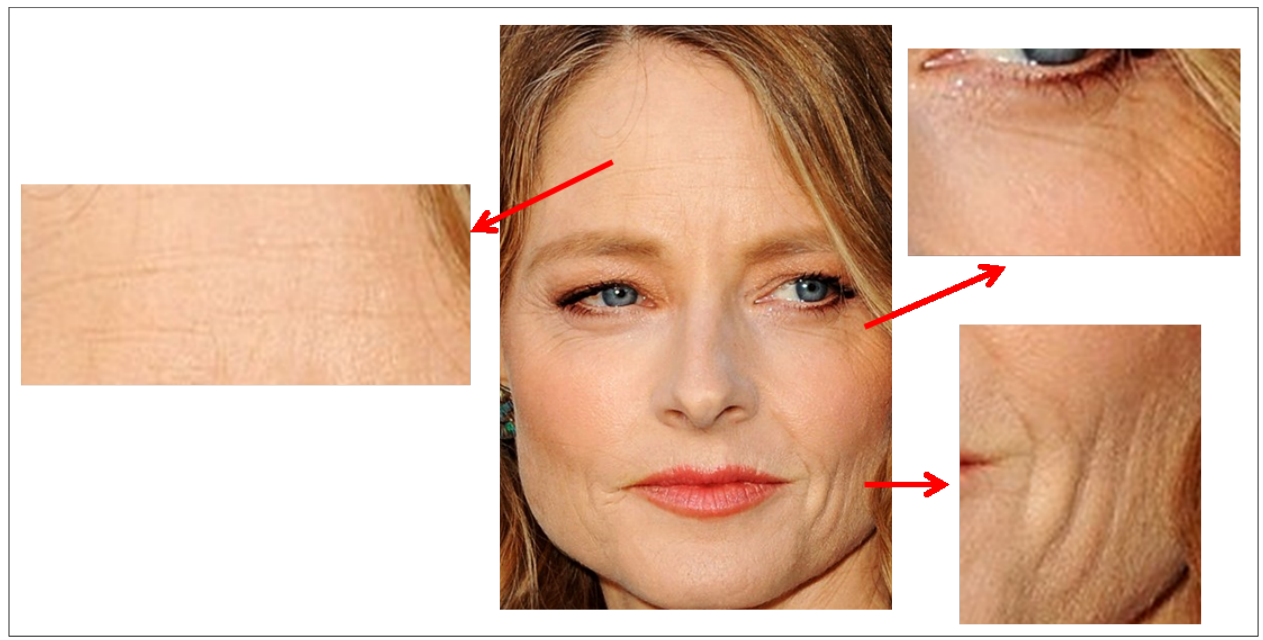

Figure 11: Image of a subject having very deep wrinkles around eyes and mouth and light wrinkles on forehead.

are the output of the algorithm, the original wrinkles are those hand-drawn by a user and the well-localized wrinkles are the wrinkles detected at correct locations. The evaluation protocol calculated quantitative results in the form of detection, false alarm and miss ratios. However, the following observations were made regarding limitations of this evaluation setup.

(A): High detection rate did not ensure results of visually high quality where the term 'quality' can be described as the continuity of a wrinkle curve as well as its smooth curvature looking more similar to real wrinkles to a user.

(B): The hand-drawn wrinkles can be subjective depending on the user perception of wrinkles. We observed that one user tended to draw more 

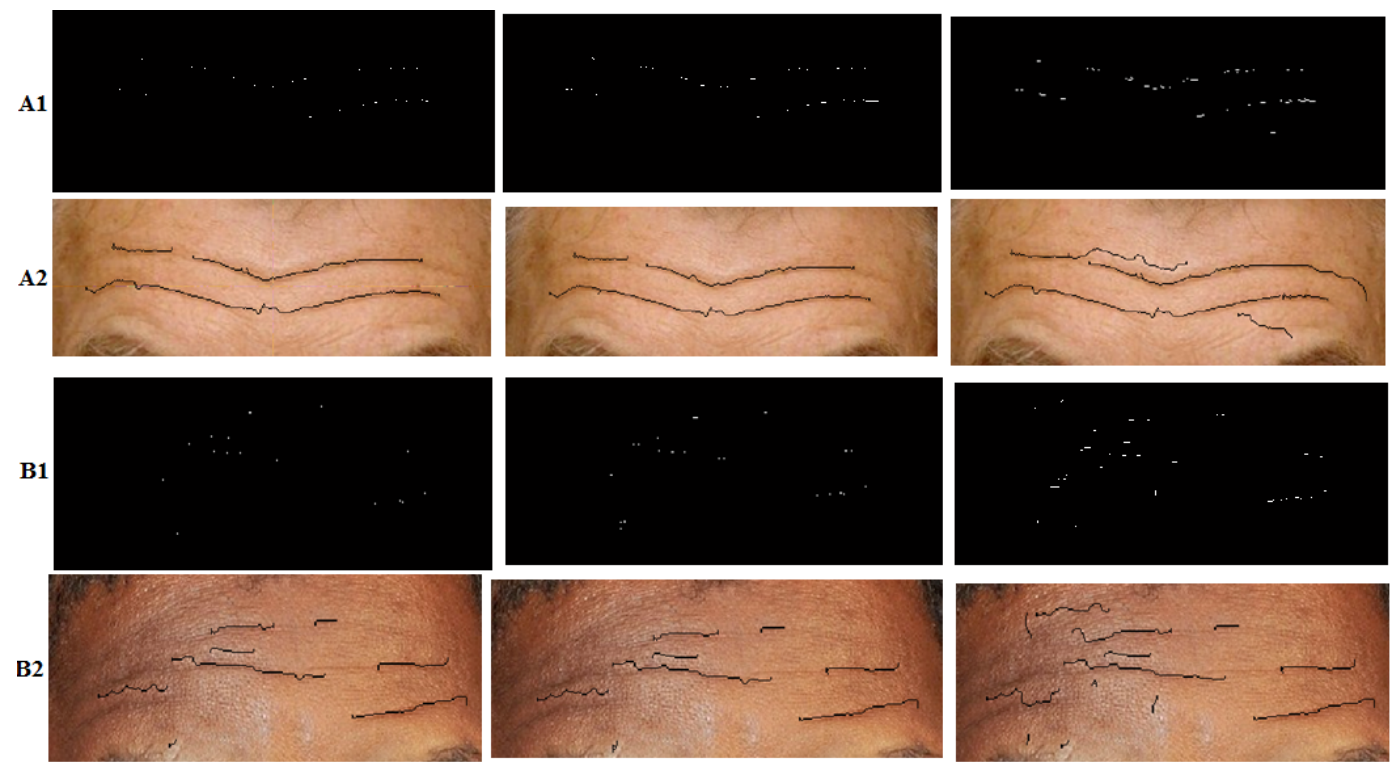

(a)

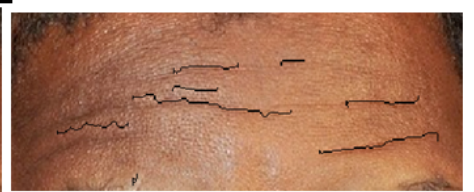

(b)

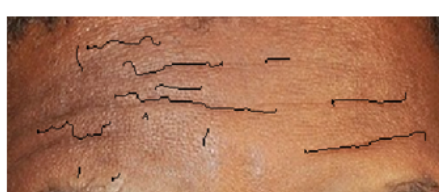

(c)

Figure 12: Effect of quantization by choosing different values of $\Delta_{t}$. (a) $\Delta_{t}=0.01$ (b) $\Delta_{t}=0.05$ (c) $\Delta_{t}=0.09$. Rows 'A1' and 'A2' represent results of an image with detection rate of greater than $70 \%$ and rows 'B1' and 'B2' represent results of an image with detection rate of $61 \%$.

wrinkles than others. This introduced subjectivity in the quantitative results calculated by our evaluation setup.

Therefore, although we follow the same evaluation set up for comparison with the previous results of MPP modeling, we make additional remarks about the quality of results when appropriate. In general, we observed that a false alarm rate lower than $0.2 \%$ contributed to visually better detection results.

\subsection{Detection Results in Medium Resolution Images}

In this experiment we downloaded images of high resolution, more than ' 1024 x 768' pixels, of public figures. There were 123 images of 60 subjects of both genders and different ethnicities and age. There were one or more images of a subject depending on quality of the images available on the Internet for that particular subject. In each image, the face was hand cropped and resized so that the maximum dimension was equal to 700 pixels. These images presented a variety of appearances of wrinkles. After running experiments, we separated 18 images with low detection results to highlight the 


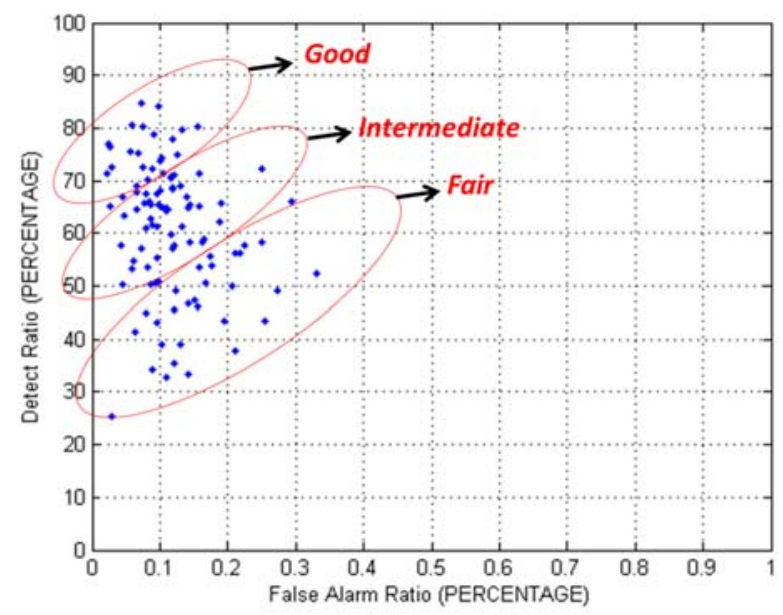

Figure 13: A plot of false alarm vs. detection rates in percentage for the 105 images of medium resolution.

typical challenges faced by a detection algorithm. We plotted the detection and false alarm ratios for the rest of 105 images shown in Figure 13. The detection rate varied from $25 \%$ to $85 \%$ with the typical rate in the range of $60-75 \%$ whereas the false alarm rate varied from $0.01 \%$ to $0.33 \%$ with the typical rate in the range of $0.05-0.15 \%$. Based on the visual observation of images, qualitatively, we sorted images in three different categories of having good, intermediate or fair visual results where low false alarm rate was as important as high detection rate in determining the visual quality of results. The qualitative categories of images can loosely be represented as regions in the plot in Figure 13.

Figure 14 includes a few examples of images with high detection rates. Figure 15 shows some examples of medium detection rate and Figure 16 examples of low detection rates.

\subsubsection{Challenges}

We observed that the following factors contributed to the degradation of localization results.

Very light wrinkles: The most common reason for low detection rates was wrinkles' being very light. Figure 17 illustrates some typical examples. It can be seen in the left column of the figure that in such cases, the intensity gradients caused by the granular skin texture are comparable 

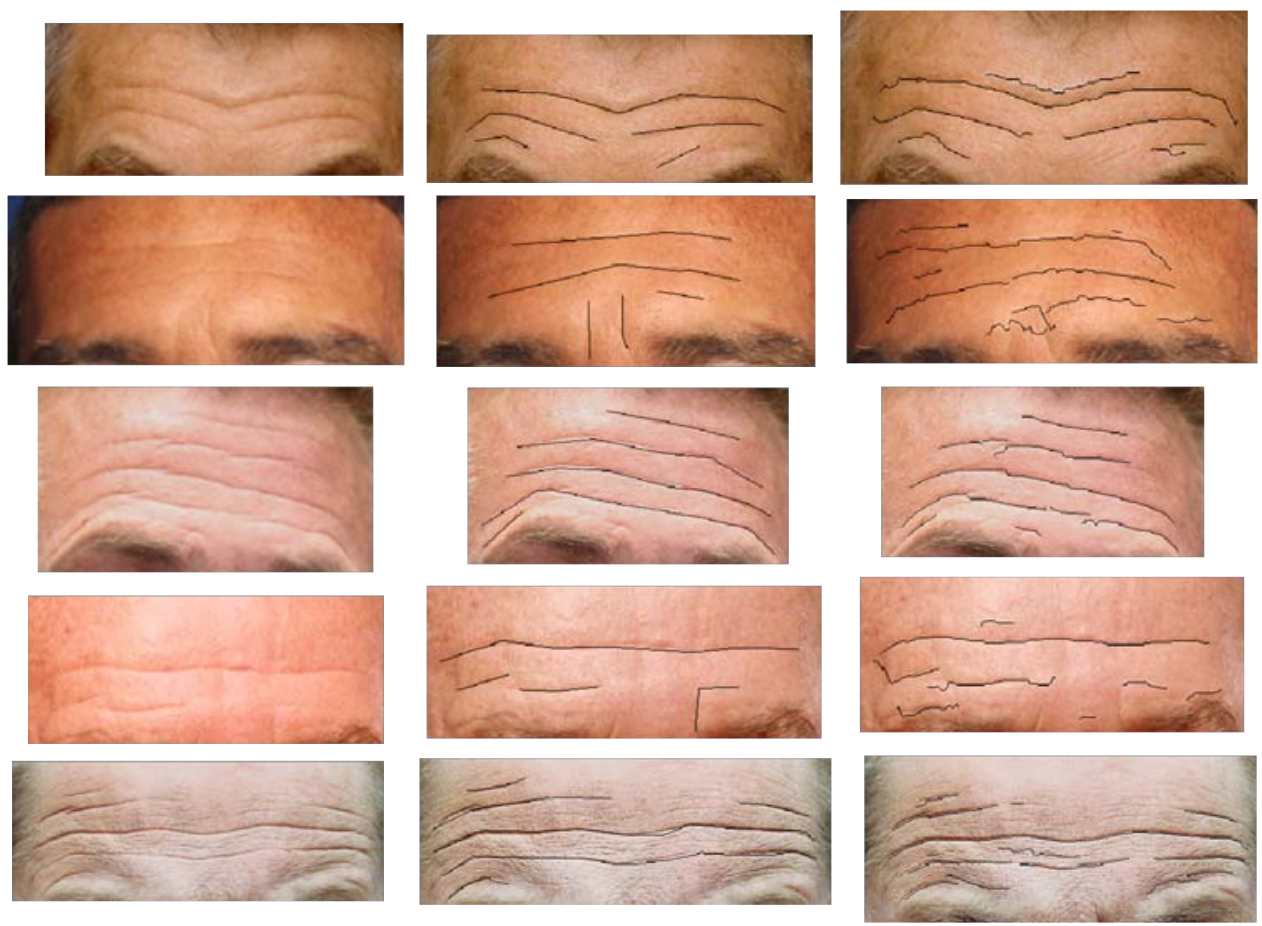

Figure 14: A few typical examples of images with a detection rate of $>70 \%$. (Left) Original (Middle) Hand-drawn (Right) Automatically Localized

to those caused by wrinkles. Consequently, the algorithm picks nonwrinkle sites as wrinkles increasing false alarm rate.

Skin discolorations: This problem was more pronounced in subjects of lighter skin color which is more prone to having moles and brown/dark spots. The intensity gradients due to the 2D skin features of discoloration are mixed with those due to the 3D skin features of wrinkles and cause erroneous maxima locations in Gabor features. Some examples are shown in Figure 18. Although our algorithm discards most of such blob-like maxima using eccentricity property of connected components, a few linear maxima are still wrongly classified as wrinkles.

Illumination and bright spots: Although we selected images with minimum illumination variation from the Internet, due to the uncontrolled acquisition settings, most of the images had some illumination variation. The variation caused 'bright spots' in images where light is reflected from 


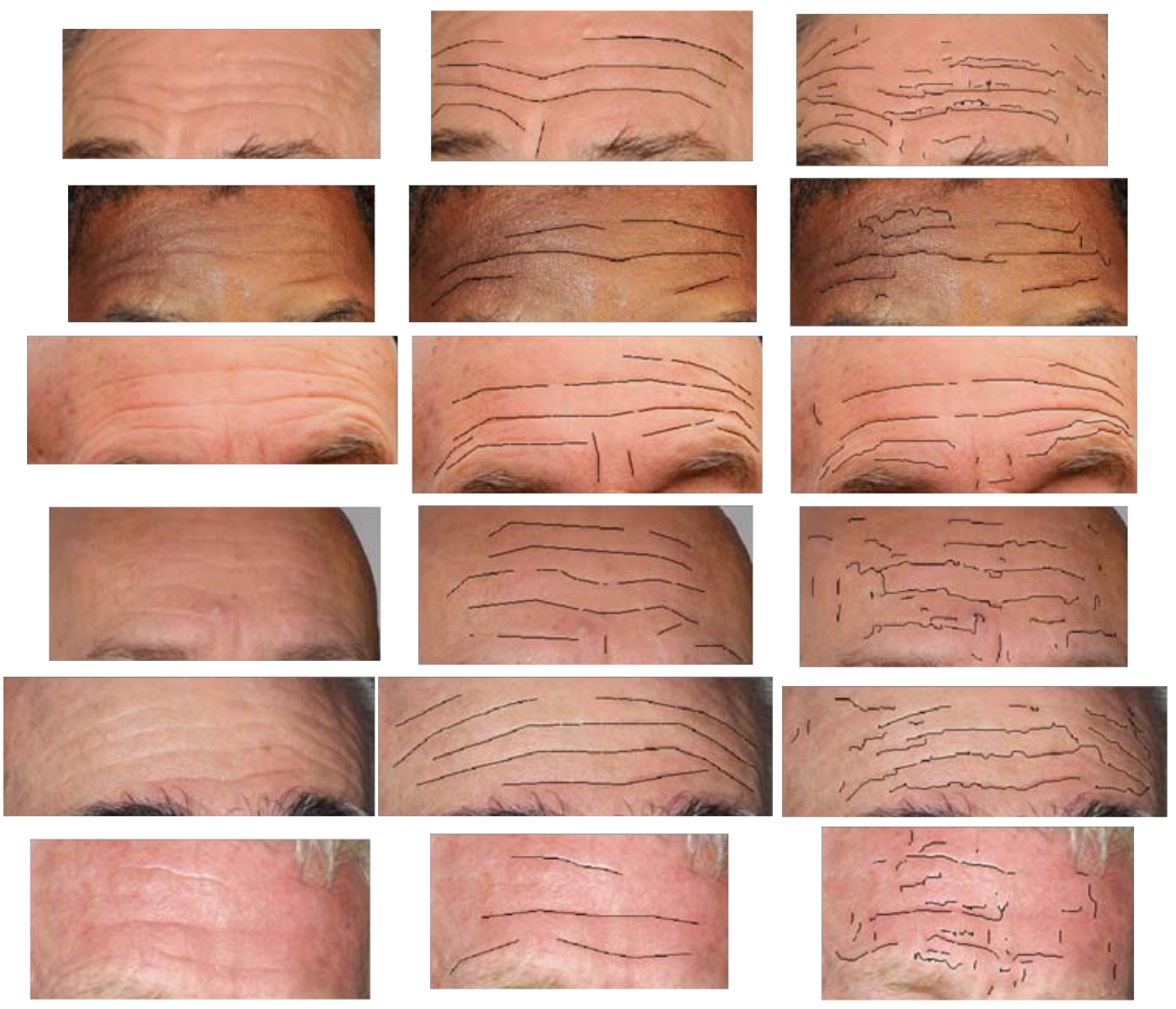

Figure 15: A few typical examples of images with a detection rate in the range of $50-65 \%$. (Left) Original (Middle) Hand-drawn (Right) Automatically Localized

skin surface due to oily skin or skin texture. In some cases, such bright spots caused false intensity gradients. Figure 19 illustrates two examples where illumination highlights irregular skin texture and causes bright spots highlighting erroneous intensity gradients in Gabor images.

\subsection{Comparison with Detection Results using MPP Modeling in Low Reso- lution Images}

Next we conducted experiments on dataset used in our previous work of detection of wrinkles using MPP modeling [9]. The dataset consists of 36 images with hand-drawn wrinkles as ground truth. The image size varies from $86 \times 37$ as minimum to $290 \times 110$ as maximum. Images were not resized in this experiment for a better comparison with MPP modeling results. There 

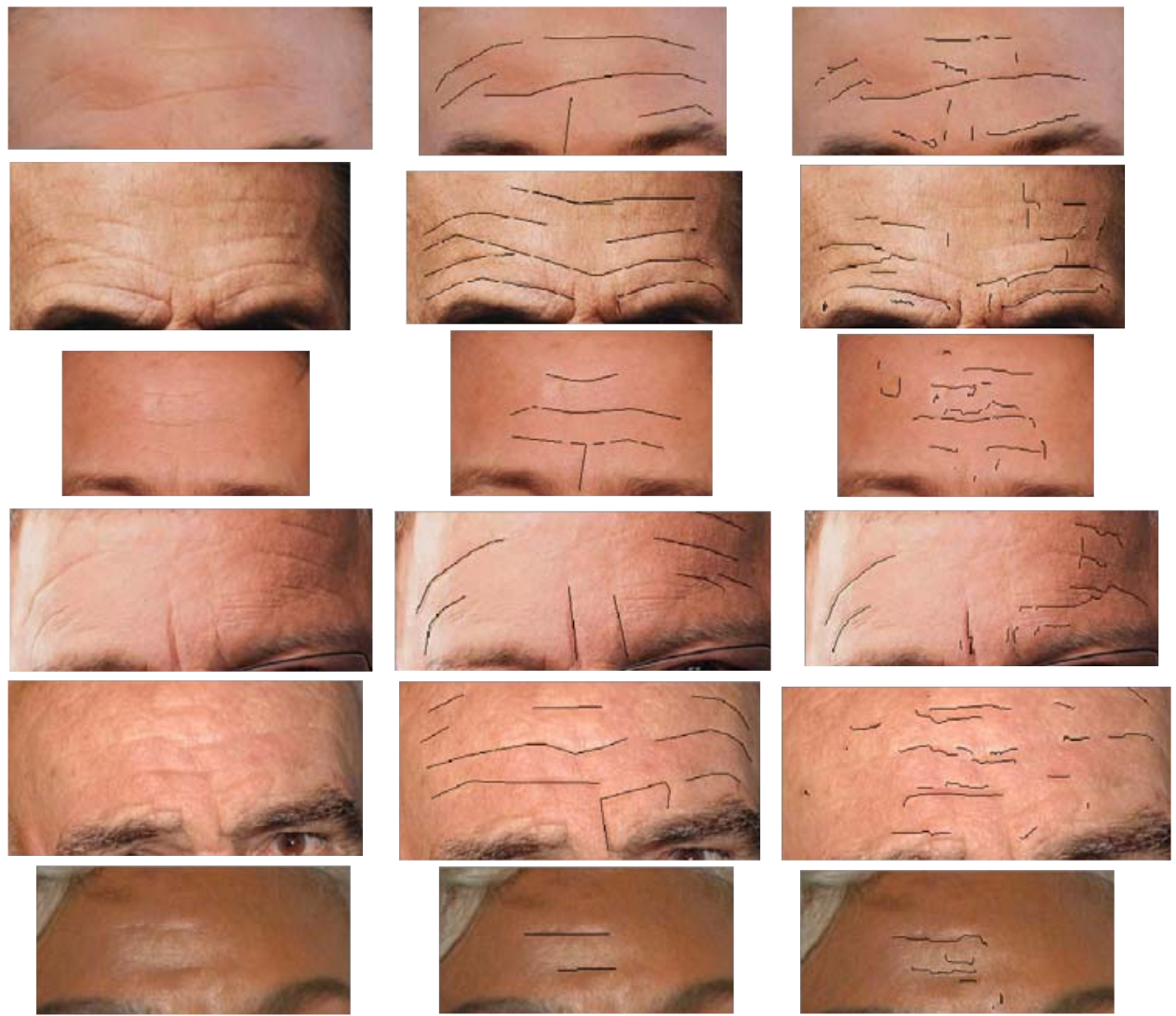

Figure 16: A few typical examples of images with a detection rate of $<50 \%$. (Left) Original (Middle) Hand-drawn (Right) Automatically Localized

was significant improvement in runtime from 65 seconds on average in case of MPP modeling to 9 seconds on average in case of current algorithm. Figure 20 shows plots of detection/false alarm rates for two methods where two distinct clusters of data points can be observed. MPP modeling classifies more image sites as wrinkles increasing both detection and false alarm rates whereas current algorithm has consistent false alarm rate around $0.01 \%$ with varying detection rate. Qualitatively, this results in visually better localization results with less false alarm rate. Figure 21 includes example images of five subjects with localization results by both approaches where the superior performance of the current algorithm can be observed. 

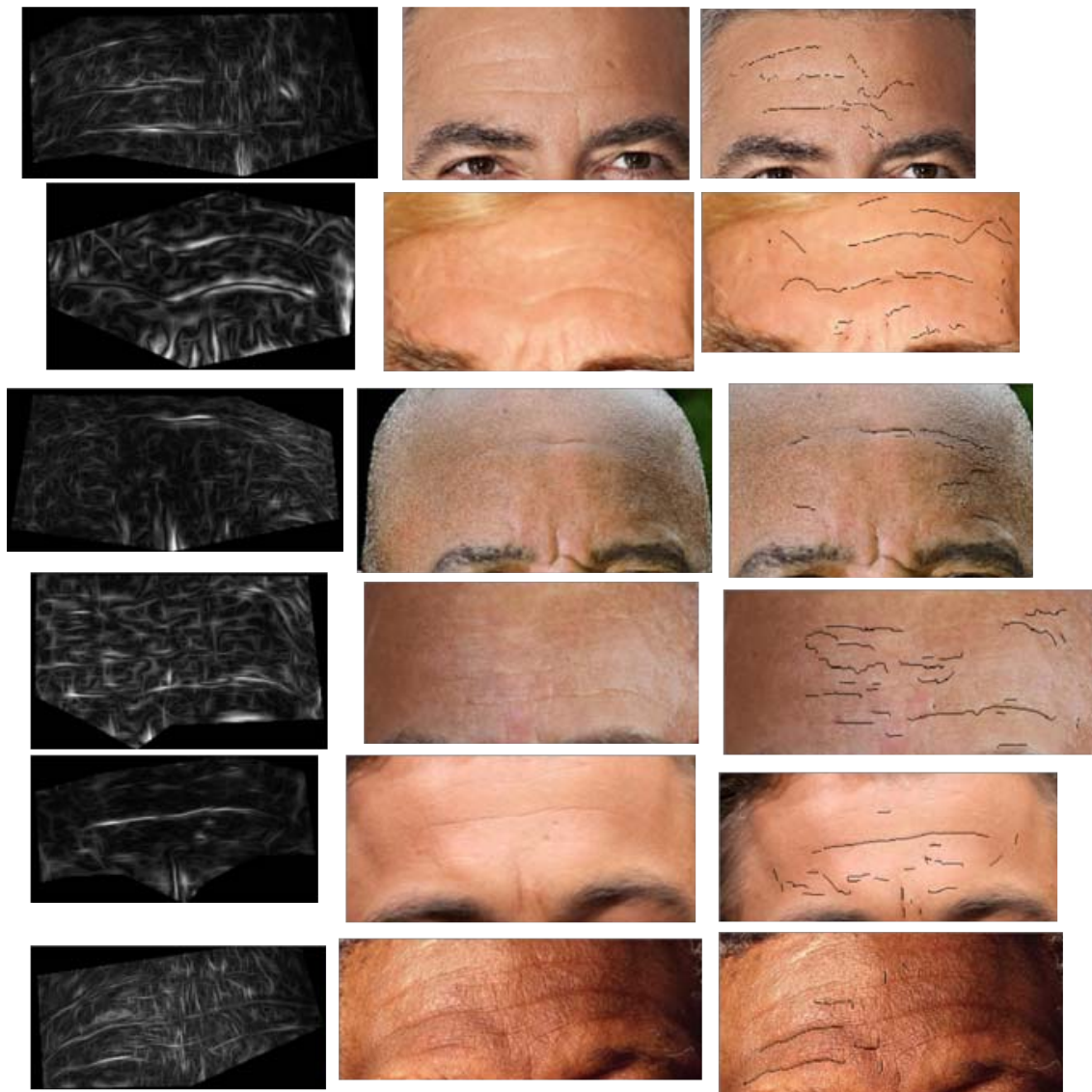

Figure 17: A few typical examples of images with very light wrinkles. (Left) Gabor features in gray scale. (Middle) Original images showing light wrinkles. (Right) Automatically localized wrinkles.

\subsection{Detection Results of FG-NET database}

We also conducted experiments on a subset of images from FG-NET database. The images consisted of subjects in old age and with relatively good quality. Scanned versions of old pictures were not included due to poor quality of skin texture in the image. Figures 22 and 23 show results for the images selected from the database along with hand-drawn wrinkles.

\subsection{Detection Results in Regions around Eyes in Medium Resolution Images}

The final set of experiments was conducted on facial region around eyes in medium resolution image. However, hand-drawn wrinkles were not available in this case and results were categorized as having high or medium detection 

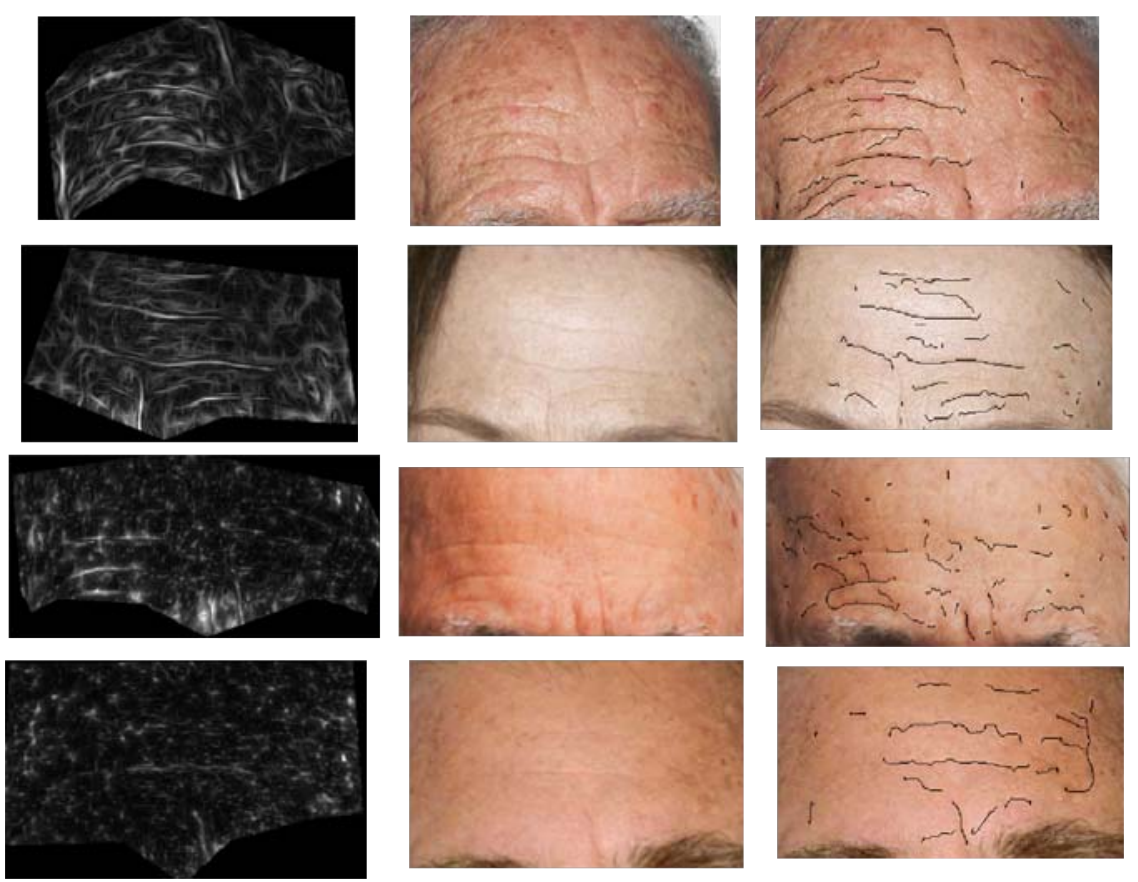

Figure 18: A few typical examples of images with skin discolorations masking wrinkles. (Left) Gabor features in gray scale. (Middle) Original images showing light wrinkles. (Right) Automatically localized wrinkles.
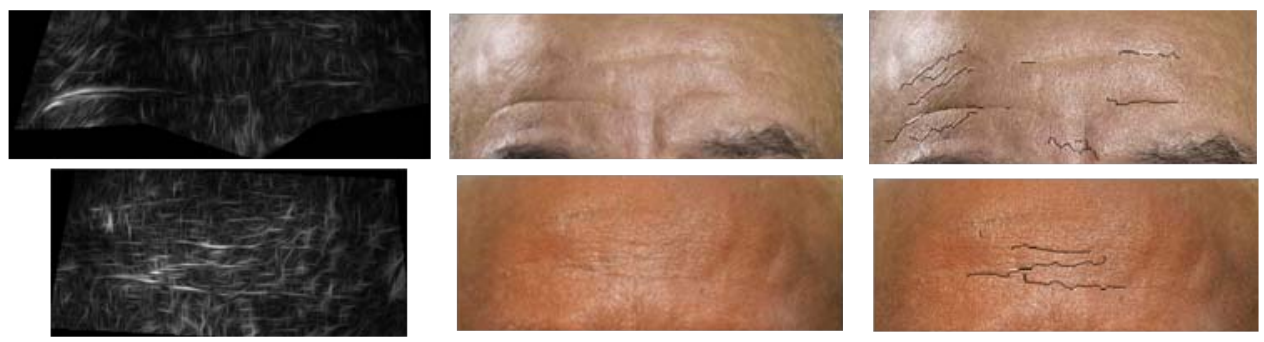

Figure 19: Two examples of images with varying illumination. (Left) Gabor features in gray scale. (Middle) Hand-drawn wrinkles. (Right) Automatically localized wrinkles.

rates by visual inspection only. Figures 24 and 25 include some examples with high detection rates and Figures 26 and 27 include examples with medium detection rates. As we observed from experimental results, facial wrinkles around eyes are different from forehead wrinkles and pose extra challenges. First, although still being curvilinear features, wrinkles around eyes are not always long and continuous. Second, most of the wrinkles around eyes are 


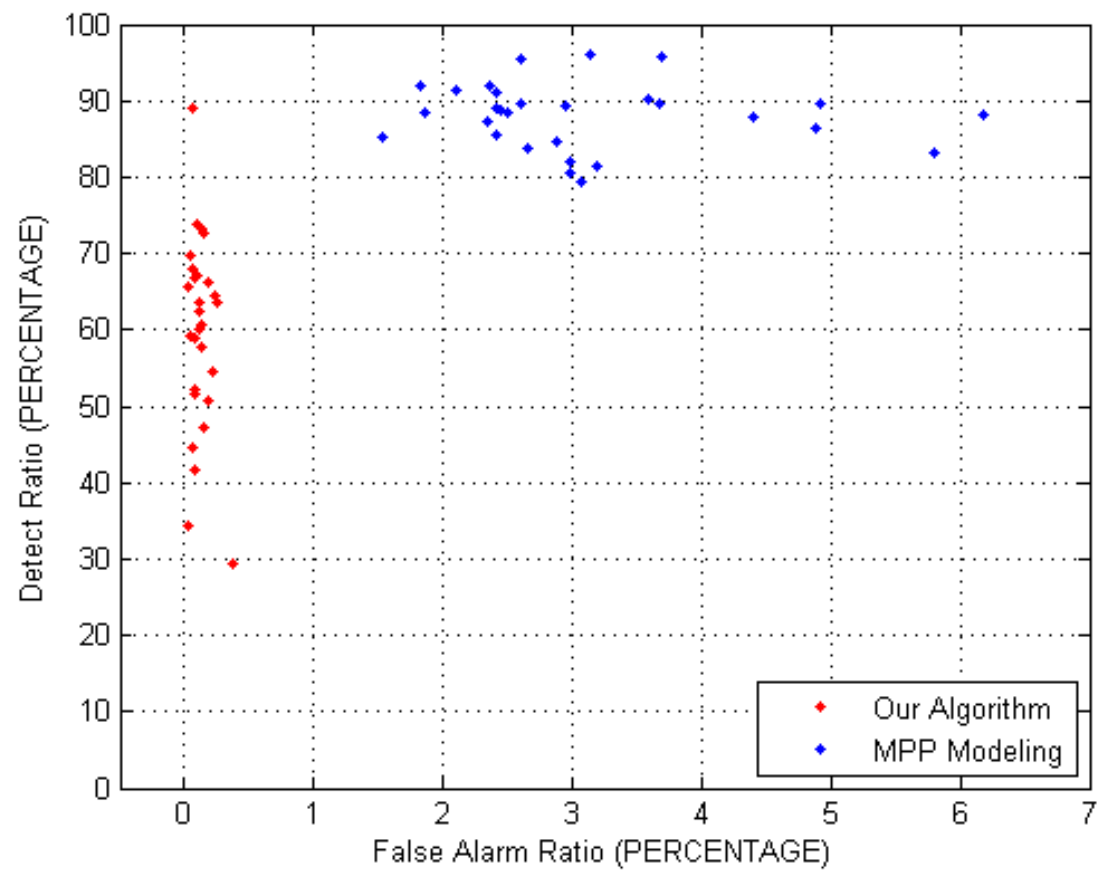

Figure 20: Plots of detection results using current algorithm and MPP modeling for low resolution images used in [9].

very light. Third, most subjects experience sagging of skin around eyes with aging. This causes a deep oval shape sag line under eye as well as granular and rough skin surface texture. As we mentioned earlier, factors like rough skin surface texture (along with illumination) and skin discoloration cause intensity gradients which interfere with those due to wrinkles and make it more challenging to localize wrinkles around eyes. Figure 28 highlights these factors in an eye image. One or more of these factors cause degradation in wrinkle localization in examples shown in Figures 26 and 27.

Finally, as is shown in Figures 24 and 25, in the absence of sagging skin or rough skin surface texture, light wrinkles are also well localized in addition to deep, continuous wrinkles. 


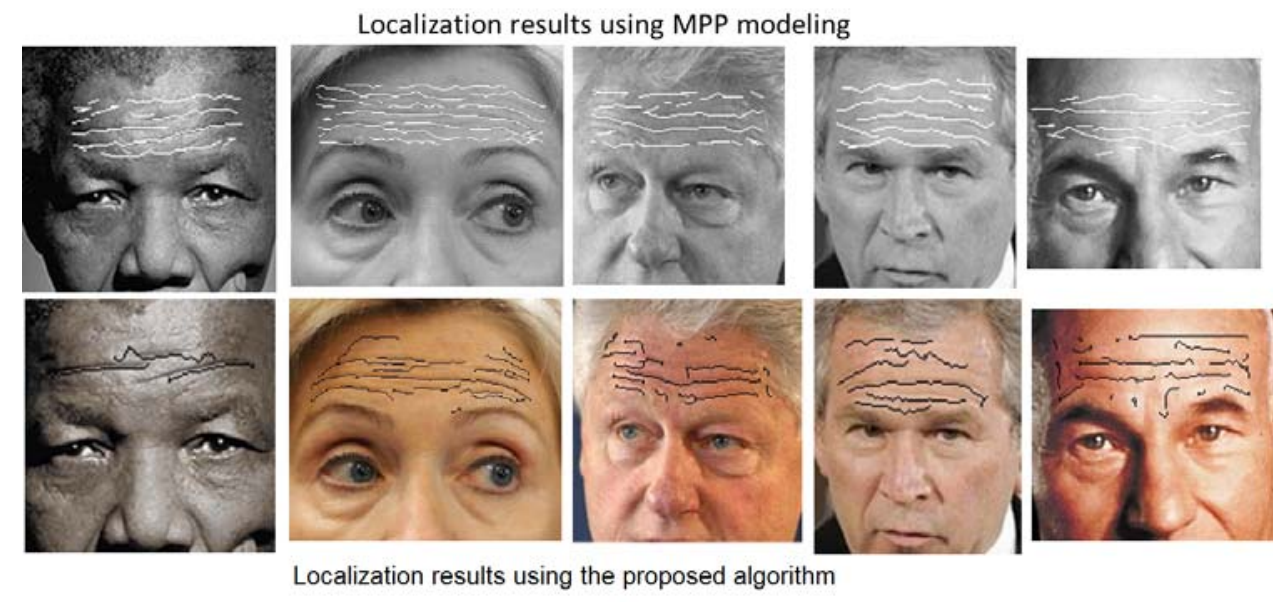

Figure 21: Visual comparison of detection results using our algorithm and MPP modeling for low resolution images used in [9].

\section{Conclusion}

In this paper, we presented a computationally efficient deterministic algorithm based on Gabor filters and image morphology to improve wrinkle localization results. We used image features based on Gabor filter bank to highlight subtle curvilinear discontinuities in skin texture caused by wrinkles and image morphology to incorporate geometric constraints of wrinkle curves. We conducted experiments on a dataset of medium resolution images downloaded from the Internet as well as a subset of FG-NET images. Experiments showed that the proposed algorithm was faster and provided better localization results by decreasing false positives. The experiments showed that wrinkles are easier to localize in subjects with smooth skin texture as compared to subjects with rough skin texture or skin discolorations.

\section{Acknowledgments}

The first author would like to acknowledge the support of Fulbright/ HEC(Pakistan)/USAID PhD Scholarship. However, the contents of this publication have not been approved by the representing agencies/governments of the USA/Pakistan. In addition, this work was partially supported by a MURI Grant N00014-08- 1-0638 from the Office of Naval Research, USA. 

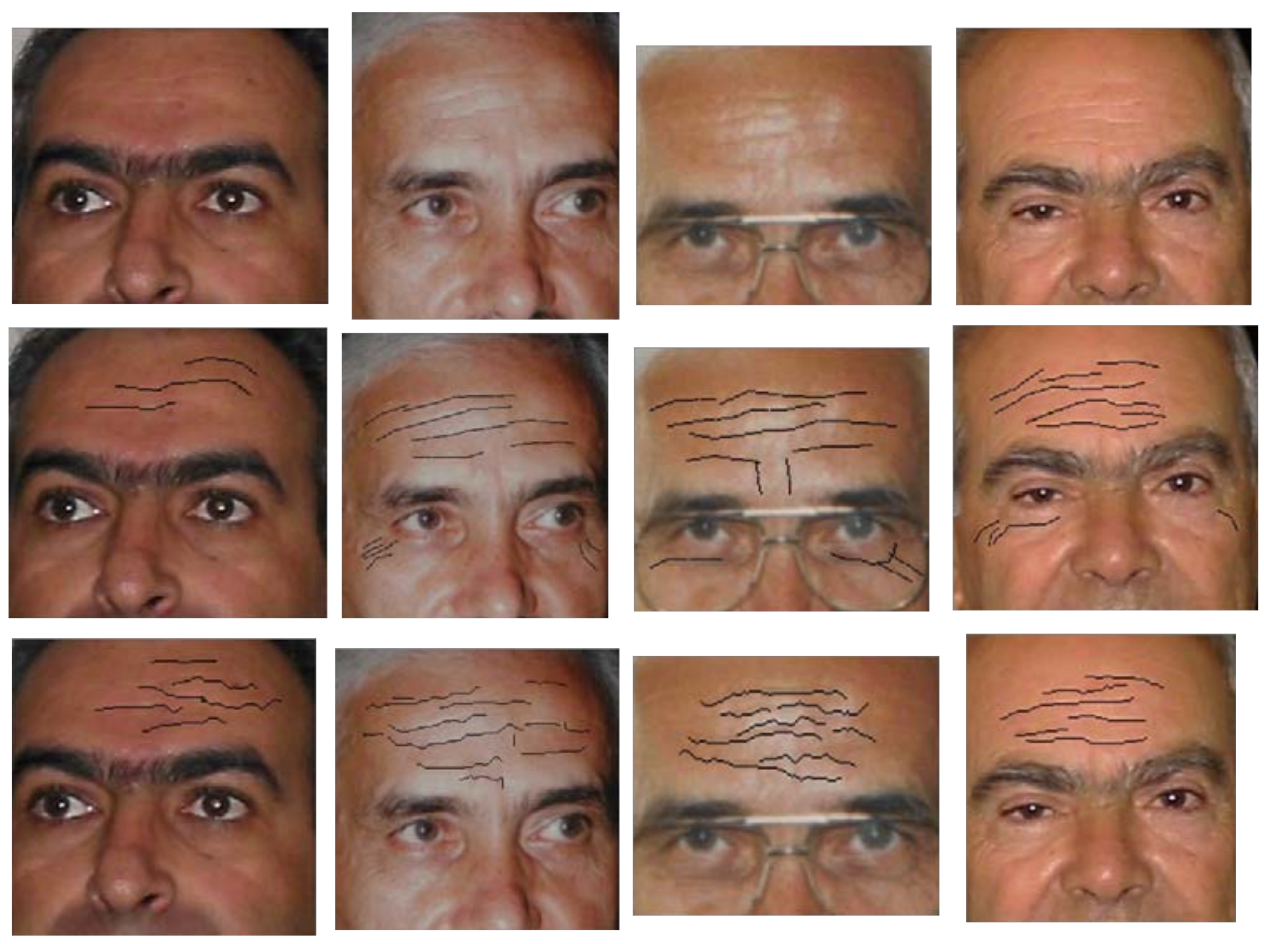

Figure 22: Localization results in FG-NET database. (Top) Original (Middle) Hand-drawn wrinkles (Bottom) Detected wrinkles. 

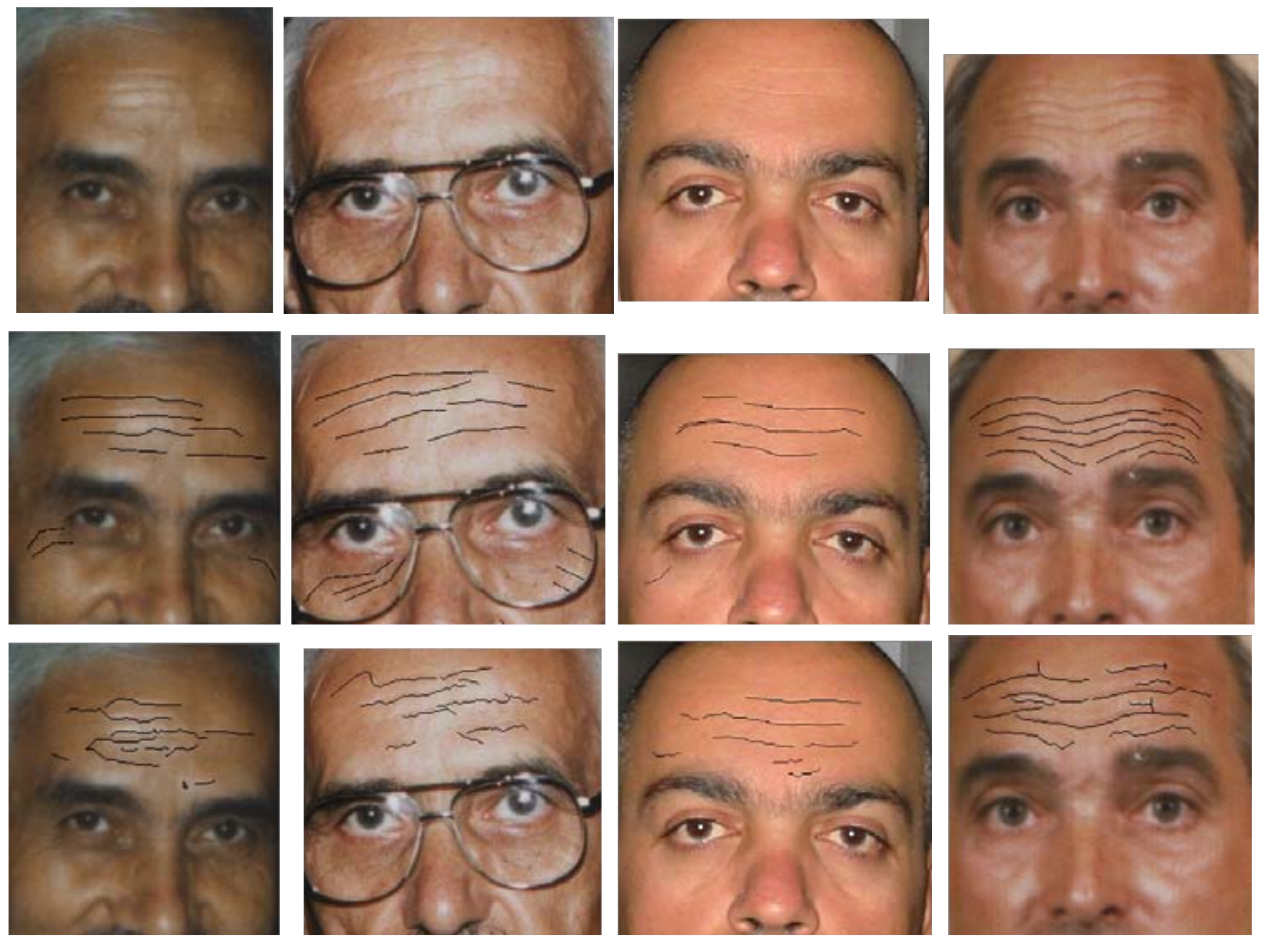

Figure 23: Localization results in FG-NET database. (Top) Original (Middle) Hand-drawn wrinkles (Bottom) Detected wrinkles.
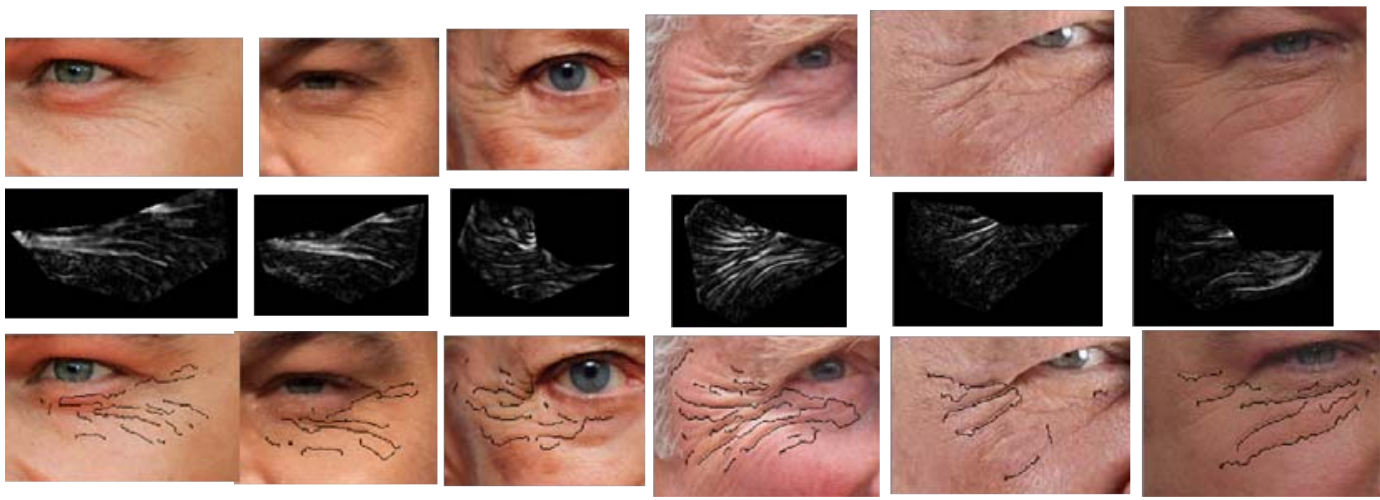

Figure 24: Examples of results with high detection rates for localization of wrinkles around eyes. (Top) Original (Middle) Gabor features in gray scale image. (Bottom) Detected wrinkles. 

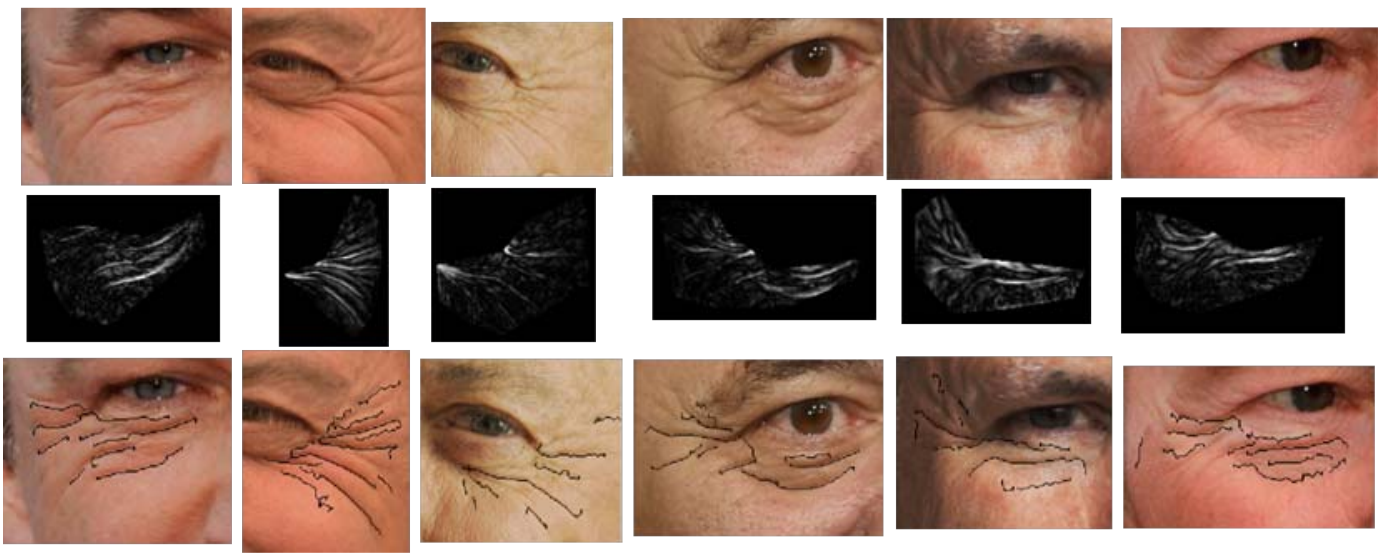

Figure 25: Examples of results with high detection rates for localization of wrinkles around eyes. (Top) Original (Middle) Gabor features in gray scale image. (Bottom) Detected wrinkles.
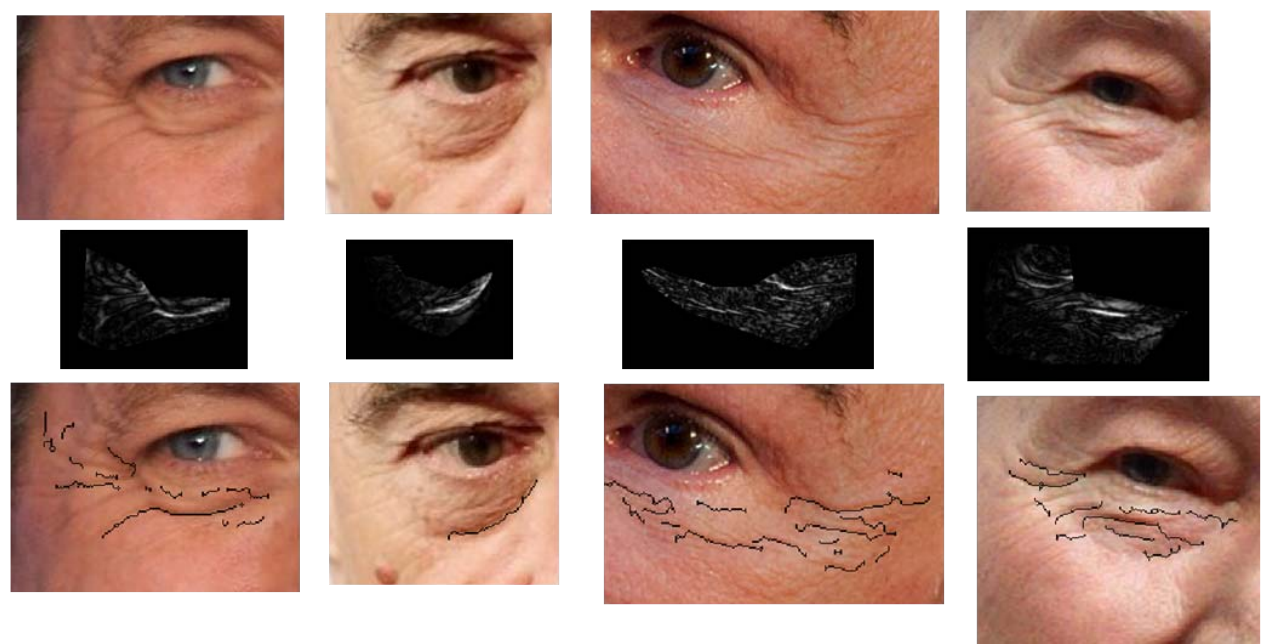

Figure 26: Examples of results with medium detection rates for localization of wrinkles around eyes. (Top) Original (Middle) Gabor features in gray scale image. (Bottom) Detected wrinkles. 

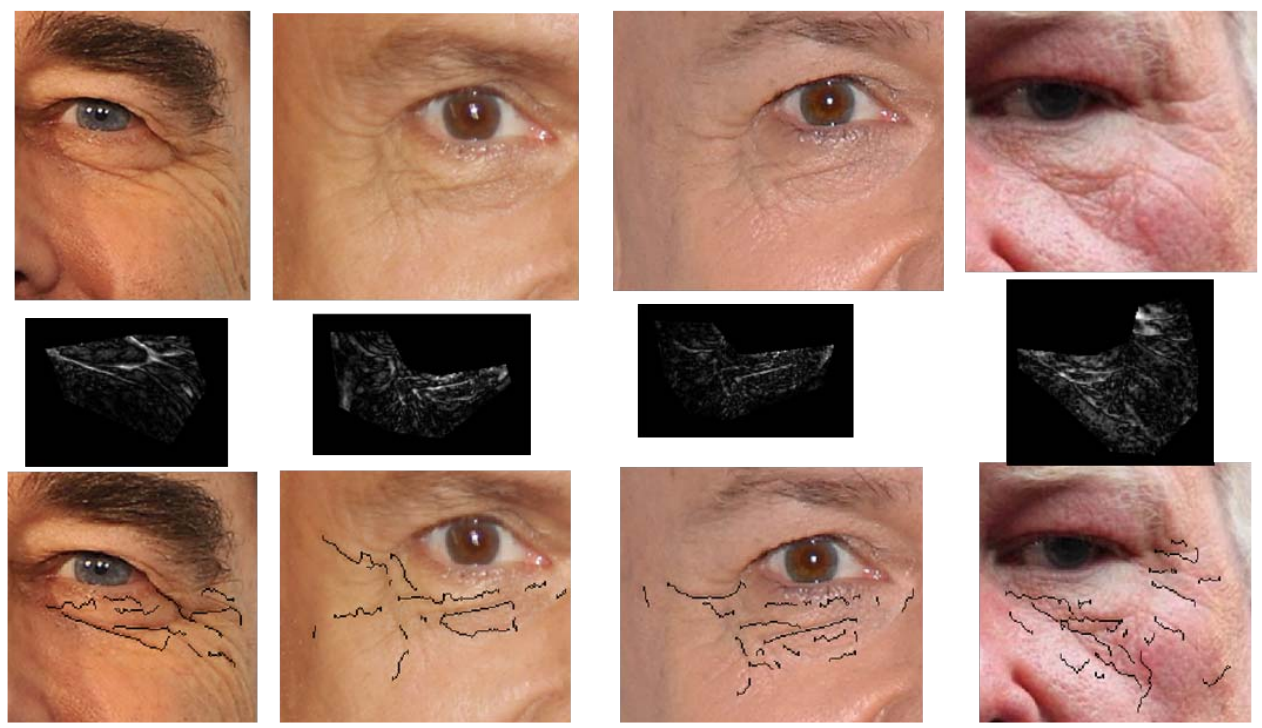

Figure 27: Examples of results with medium detection rates for localization of wrinkles around eyes. (Top) Original (Middle) Gabor features in gray scale image. (Bottom) Detected wrinkles.
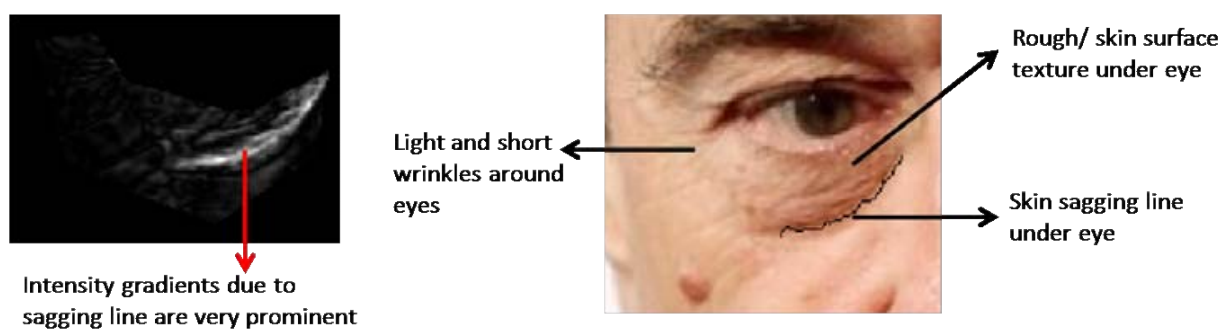

Figure 28: Typical challenges in detection of wrinkles around eyes i.e. under-eye sagging and very light wrinkles. 


\section{References}

[1] Y. Fu, G. Guo, T. Huang, Age synthesis and estimation via faces: A survey, PAMI 32 (11) (2010) 1955-1976.

[2] K. Luu, T. D. Bui, C. Y. Suen, K. Ricanek, Combined local and holistic facial features for age-determination, in: ICCARV, 2010, pp. 900-904.

[3] J. Suo, F. Min, S. Zhu, S. Shan, X. Chen, A multi-resolution dynamic model for face aging simulation, in: CVPR, 2007, pp. 1-8.

[4] N. Ramanathan, R. Chellappa, S. Biswas, Computational methods for modeling facial aging: A survey, J. Vis. Lang. Comput. 20 (3) (2009) $131-144$.

[5] N. Ramanathan, R. Chellappa, Modeling shape and textural variations in aging faces, in: FG, 2008, pp. 1-8.

[6] Y. H. Kwon, N. D. V. Lobo, Age classification from facial images, in: CVPR, 1999.

[7] G. O. Cula, P. R. Bargo, N. Kollias, Assessing facial wrinkles: automatic detection and quantification (2009).

[8] N. Batool, R. Chellappa, Modeling and detection of wrinkles in aging human faces using marked point processes, in: ECCV Workshops (2), 2012, pp. 178-188.

[9] N. Batool, R. Chellappa, A Markov point process model for wrinkles in human faces, in: ICIP, 2012, pp. 1809-1812.

[10] R. Stoica, X. Descombes, J. Zerubia, A Gibbs point process for road extraction from remotely sensed images, IJCV 57 (2004) 121-136.

[11] O. G. Cula, K. J. Dana, F. P. Murphy, B. K. Rao, Skin texture modeling, IJCV 62 (2005) 97-119.

[12] N. Batool, S. Taheri, R. Chellappa, Assessment of facial wrinkles as a soft biometrics, in: FG, 2013, pp. 1-7.

[13] N. Batool, R. Chellappa, Detection and inpainting of facial wrinkles using texture orientation fields and Markov random field modeling, IEEE Trans. on Image Processing (in print). 
[14] S. Chambon, J.-M. Moliard, Automatic road pavement assessment with image processing: Review and comparison, International Journal of Geophysics 2011 (2011) 20.

[15] M. M. Fraz, P. Remagnino, A. Hoppe, B. Uyyanonvara, A. R. Rudnicka, C. G. Owen, S. A. Barman, Blood vessel segmentation methodologies in retinal images - a survey, Comput. Methods Prog. Biomed. 108 (1) (2012) 407-433.

[16] F. J. Ayres, R. M. Rangayyan, Design and performance analysis of oriented feature detectors, Journal of Electronic Imaging 16 (2) (2007) 023007-023007-12.

[17] F. Zana, J.-C. Klein, Segmentation of vessel-like patterns using mathematical morphology and curvature evaluation, Image Processing, IEEE Transactions on 10 (7) (2001) 1010-1019.

[18] A. Landstrom, M. Thurley, Morphology-based crack detection for steel slabs, Selected Topics in Signal Processing, IEEE Journal of 6 (7) (2012) 866-875.

[19] B. Lam, H. Yan, A novel vessel segmentation algorithm for pathological retina images based on the divergence of vector fields, Medical Imaging, IEEE Transactions on 27 (2).

[20] T. Lelore, F. Bouchara, Fair: A fast algorithm for document image restoration, Pattern Analysis and Machine Intelligence, IEEE Transactions on 35 (8) (2013) 2039-2048. 